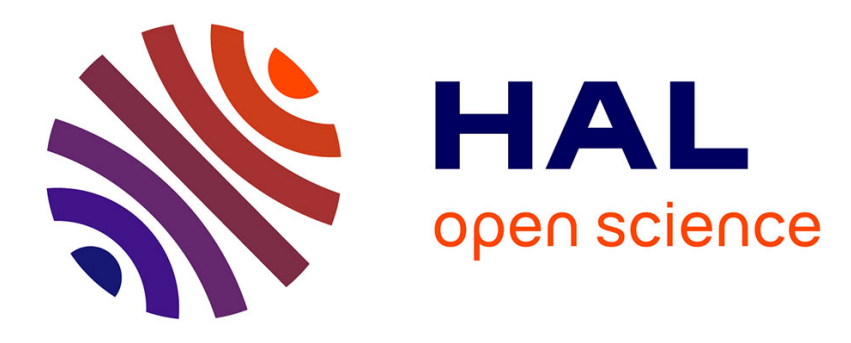

\title{
Solvability of the halting and reachability problem for binary 2-tag systems
}

Liesbeth de Mol

\section{To cite this version:}

Liesbeth de Mol. Solvability of the halting and reachability problem for binary 2-tag systems. Fundamenta Informaticae XX, 2010, 99 (4), pp.435-471. 10.3233/FI-2010-257 . hal-01396536

\section{HAL Id: hal-01396536 \\ https://hal.univ-lille.fr/hal-01396536}

Submitted on 14 Nov 2016

HAL is a multi-disciplinary open access archive for the deposit and dissemination of scientific research documents, whether they are published or not. The documents may come from teaching and research institutions in France or abroad, or from public or private research centers.
L'archive ouverte pluridisciplinaire HAL, est destinée au dépôt et à la diffusion de documents scientifiques de niveau recherche, publiés ou non, émanant des établissements d'enseignement et de recherche français ou étrangers, des laboratoires publics ou privés. 
IOS Press

\title{
Solvability of the halting and reachability problem for binary 2-tag systems
}

\author{
Liesbeth De Mol* \\ Centre for Logic and Philosophy of Science \\ Gent University
}

Blandijnberg 2, 9000 Gent, Belgium

elizabeth.demol@ugent.be

\begin{abstract}
In this paper a detailed proof will be given of the solvability of the halting and reachability problem for binary 2 -tag systems.
\end{abstract}

Keywords: $v$-Tag systems, halting problem, reachability problem, non-universality

\section{Introduction}

\subsection{Post's frustrating problem of "tag"}

Tag systems were invented and studied by Emil Leon post $[15,16]$ during his Procter fellowship in mathematics at Princeton during the academic year 1920-21. They played an important role in his work on normal systems, which he also developed during that time, and led to the reversal of his program to prove the recursive solvability of the Entscheidungsproblem for first-order predicate calculus. Indeed, after 9 months of intensive research on tag systems, Post first came to the conclusion that proving the decidability of the Entscheidungsproblem might be impossible. He never proved that this decision problem is recursively unsolvable. This was done by Church and Turing in their seminal papers published in 1936 $[1,19]$. However, already in 1921 he did prove that there are unsolvable decision problems for normal

Address for correspondence: Blandijnberg 2, 9000 Gent, Belgium

${ }^{*}$ This research was supported by the Fund for Scientific Research - Flanders (FWO - Vlaanderen) and the Kunsthochschule für Medien, Köln. I am very grateful to the anonymous referee who helped me to improve this paper in a very significant way. I would also like to thank M. Bullynck, A. Moore and D. Devreese for their support during the time I was working on this paper and M. Davis, M. Margenstern, T. Neary and D. Woods for having stimulated me in my ongoing research on tag systems. 
systems, on the basis of a thesis similar to Church's and Turing's, called Post's thesis [6, 7]. Unfortunately, he never made any attempt to publish these results at that time. Later, in the forties, he provided a detailed description of his results from the period 1920-1921 in his Absolutely unsolvable problems and relatively undecidable propositions - Account of an anticipation [16], a posthumously published manuscript edited by Martin Davis. More detailed information on these more historical matters can be found in $[6,7,8,18]$.

Definition 1. (v-tag system) A tag system $T$ consists of a finite alphabet $\Sigma$ of $\mu$ symbols, a deletion number $v \in \mathbb{N}$ and a finite set of $\mu$ words $w_{0}, w_{1}, \ldots, w_{\mu-1} \in \Sigma^{*}$ called the appendants, where any appendant $w_{i}$ corresponds to $a_{i} \in \Sigma$. A $v$-tag system has a deletion number $v$.

In a computation step of a tag system $T$ on a word $A \in \Sigma^{*}, T$ appends the appendant associated with the leftmost letter of $A$ at the end of $A$, and deletes the first $v$ symbols of $A .{ }^{1}$ This computational process is iterated until the tag system produces the empty word $\epsilon$. Note that tag systems are monogenic and thus deterministic. Following the notation of [21], $A_{i} \vdash A_{i+1}$ means that $A_{i+1}$ is produced from $A_{i}$ after one computation step, $A_{i} \vdash^{n} A_{i+n}$ that $A_{i+n}$ is produced after $n$ computation steps from $A_{i}$.

To give an example, let us consider the one tag system mentioned by Post with $v=3,0 \rightarrow 00,1 \rightarrow 1101$ $[15,16]$. If the initial word $A_{0}=110111010000$ we get the following productions:

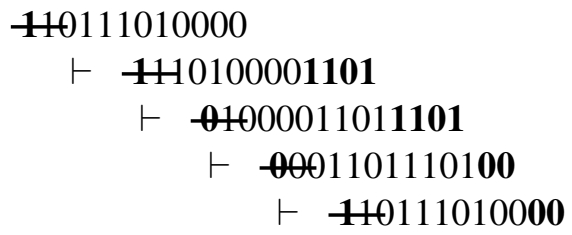

The word $A_{0}$ is reproduced after 4 computation steps and is thus an example of a periodic word. Post called the behavior of this one tag system "intractable". Up to now, it is still not known whether this particular example is recursively solvable, despite its formal simplicity. Post also mentioned that he studied the class of tag systems with $v=2, \mu=3$ and described this class as being of "bewildering complexity" and as "[...] leading to an overwhelming confusion of classes of cases, with the solution of the corresponding problem depending more and more on problems of ordinary number theory." [16]. Post identified three classes of ultimate behavior in tag systems that will be used throughout this paper.

Definition 2. (halt) A tag system $T$ is said to halt on an initial word $A_{0}$ when there is an $n \in \mathbb{N}$ such that $T$ produces the empty word $\epsilon$ after $n$ computation steps on $A_{0}$, i.e., $A_{0} \vdash^{n} \epsilon$ in $T$.

Definition 3. (periodicity) A tag system $T$ is said to become periodic on an initial word $A_{0}$ if there are $n, p \in \mathbb{N}$ such that $A_{0} \vdash^{n} A_{n}$ and $A_{n} \vdash^{p} A_{n+p}=A_{n}$ in $T$. $A_{n}$ is said to be a periodic word in $T$ with period $p$.

\footnotetext{
${ }^{1}$ It should be noted that we follow Post's original definition of tag systems, instead of the one that is now commonly used. I.e. instead of first deleting the first $v$ letters and then tagging the appendant, an appendant is first tagged and then the first $v$ symbols are deleted. As a consequence, a tag system will not necessarily halt on a given word when its length has become smaller than $v$. The proof of the main Theorem only needs some minor changes to be applicable for this slightly different definition.
} 
Definition 4. (unbounded growth) A tag system $T$ is said to have unbounded growth on an initial word $A_{0}$, if for every number $n \in \mathbb{N}$ there is an $i \in \mathbb{N}$ such that for every number $j>i$, any word $A_{j}$, $A_{0} \vdash^{j} A_{j}, l_{A_{j}}>n$.

Post considered two decision problems for tag systems, which we will call the halting problem and the reachability problem for tag systems.

Definition 5. (halting problem) The halting problem for tag systems is the problem to determine for a given tag system $T$ and any initial word $A_{0}$ whether or not $T$ will halt on $A_{0}$.

Definition 6. (reachability problem) The reachability problem for tag systems is the problem to determine for a given tag system $T$, a fixed initial word $A_{0}$ and an arbitrary word $A \in \Sigma^{*}$, whether or not there is an $n$ such that $A_{0} \vdash^{n} A$ in $T$.

Note that the halting problem is a special case of the reachability problem.

Post never proved that tag systems are recursively unsolvable. It was Minsky who proved the result in 1961 [13], after the problem was suggested to him by Martin Davis, who was a student of Post. He showed that any Turing machine can be reduced to a tag system with $v=6$. The result was improved by Cocke and Minsky $[2,3,14]$. They proved that any Turing machine can be reduced to a tag system with $v=2$. Maslov generalized this result and proved that for any $v>1$ there exists at least one tag system with an unsolvable decision problem and furthermore proved that any tag system for which $v=1$ has a solvable reachability problem [12]. This last result was also proven independently by Wang [20].

Both $\mu$ and $v$ can be regarded as decidability criteria [11] for tag systems, since their solvability depends on the size of these parameters. Another such criterion is the length of the appendants. Wang proved that any tag system for which $l_{\min } \geq v$ or $l_{\max } \leq v$ has a solvable halting and reachability problem [20].

Post mentions that he proved the solvability of the reachability problem (and thus also of the halting problem) for the class of 2-tag systems with $\mu=2$, but, regretfully, never published the proof. However, he does mention that he used the three classes of ultimate behavior in tag systems he had found. Given any tag system $T$ with $\mu=v=2$ and any initial word $A_{0}$, Post was able to prove that one can decide in a finite number of steps whether or not $T$ will have unbounded growth on $A_{0}$. Clearly, if one can determine for any such $T$ and any initial word $A_{0}$ it operates on what its ultimate behavior will be one immediately gets that the reachability problem for this class of tag systems is decidable. A proof of this result has recently been found. An outline of this proof can be found in [9]. In this paper we will present the details of this proof.

\subsection{Preliminaries}

In the remainder of the paper we will use the notations and definitions given in this paragraph.

Let $T$ be a $v$-tag system in the class of tag systems $\operatorname{TS}(v, \mu)$ with a deletion number $v, \mu$ symbols and appendants $w_{0}, w_{1}, \ldots, w_{\mu-1}$. Then:

a. $l_{A}$ denotes the length of the word $A$.

b. $a^{n}$ means that $a$ is repeated $n$ times.

c. $l_{\text {max }}$ denotes the length of the lengthiest appendant $w_{i}, l_{\min }$ the length of the shortest appendant $w_{j}$, $0 \leq i, j<\mu$. 
d. $\# a_{i}$ denotes the total number of occurrences of the symbol $a_{i}$ in the appendants $w_{0}, \ldots w_{\mu-1}$.

e. An odd number is denoted as $\dot{x}$, an even number as $\ddot{x}$. If a number $x$ can be either even or odd, it is denoted as $x$.

f. $\lfloor x / y\rfloor$ is the largest integer $\leq x / y,\lceil x / y\rceil$ is the smallest integer $\geq x / y,[x / y]$ denotes either $\lfloor x / y\rfloor$ or $\lceil x / y\rceil$

g. Given some word $W \in \Sigma$ then $W^{-}$is $W$ minus its leftmost letter.

h. Given a word $A=a_{0} a_{1} \ldots a_{l_{A}-1}$, we will say that $A$ is entered with shift $x$ by $T$, when $T$ erases $a_{0} . . a_{x-1}$ and the first symbol read in $A$ by $T$ is $a_{x}$.

i. A round of $T$ on a word $A$ is a number of $\left\lceil l_{A} / v\right\rceil$ computation steps of $T$ on $A$. Note that one round of $T$ on $A$ is exactly the smallest number of computation steps that result in all the letters of $A$ being deleted by $T$ [20]. For any initial word $A_{0}$ the word produced after $j$ rounds on $A_{0}$ will be written as $Q_{j}$. j. An $s$-round of $T$ on a word $A=a_{0} a_{1} \ldots a_{l_{A}-1}$ produces the word:

$$
A^{\prime}=w_{a_{s}} w_{a_{v+s}} w_{a_{2 v+s}} \ldots w_{a_{v\left(\left\lceil A_{A}-s / v\right\rceil\right)-3+s}}
$$

The word $A^{\prime}$ is thus the result of one round of $T$ on $a_{s} a_{s+1} a_{s+2} \ldots a_{l_{A}-1}(A$ entered with shift $s$ ) without its first $\overline{\left(l_{A}-s \bmod v\right)}$ letters being deleted. I.e., the result of concatenating the appendants associated with every letter read in $A$ when entered with shift $s$. To explain this with an example, let $T$ be the example provided by Post (Sec. 1.1) and $A=\mathbf{1 1} \underline{011} \underline{101}$, then the result of one 0-round on $A$ is the word $A^{\prime}=1101110100$, the result of one 2-round on $A$ the word $A^{\prime}=001101$. Note that an $s$-round on $A$ gives the same result as a round on $A$ if $s=0$ and $l_{A} \equiv 0 \bmod v$.

$\mathrm{k}$. The additive complement $\overline{(x \bmod y)}$ of a given number $x$ relative to a modulus $y$ is defined as follows:

$$
\overline{(x \bmod y)}= \begin{cases}y-(x \bmod y) & \text { if } x \neq 0 \bmod y \\ 0 & \text { if } x \equiv 0 \bmod y\end{cases}
$$

\section{Solvability of the Halting and Reachability Problem of the Class TS $(2,2)$}

In [16] Post remarks that his proof of the solvability of the reachability problem of the class TS $(2,2)$ involved "considerable labor". This is also true for the proof we have been able to establish, involving the analysis of a large number of cases. One of the major difficulties is that, contrary to classes of Turing machines $\operatorname{TM}(m, n)$, one not only has to cope with an infinite number of initial words for each tag system in TS(2,2), but one also has to reduce an infinite number of tag systems to a finite number of cases.

In the current proof Post's approach for proving the decidability of the class TS(2,2) is applied by making use of the three classes of ultimate behavior for tag systems he identified (See Sec. 1.1). I.e., it is proven that given an arbitrary tag system $T \in \mathrm{TS}(2,2)$ and any initial word $A_{0} \in\{0,1\}^{*}$, one can decide in a finite number of steps whether or not $T$ will halt, become periodic or have unbounded growth and we can thus prove the following Theorem:

Theorem 1. For any given tag system $T$, if $\mu=v=2$ then the reachability problem for $T$ is solvable.

Since the halting problem is a special case of the reachability problem, we get the following immediate corollary from Theorem 1 :

Corollary 1. For any given tag system $T$, if $\mu=v=2$ then the halting problem for $T$ is solvable. 
In the remainder of this section we will first explain the main method of the proof (Sec. 2.1) and provide an overview of the general structure of the proof and an explanation of how each of the subcases are determined. This provides an understanding of some of the fundamental differences between the subcases that need to be addressed in order to prove Theorem 1 (Sec. 2.2). We will then turn to the details of the actual proof (Sec. 2.3).

\subsection{The table method}

The basic technique of the proof is called the table method [9]. Intuitively speaking, given a $v$-tag system $T$ with alphabet $\Sigma$ and the appendants $w_{0}, \ldots, w_{\mu-1}$ this method is used to study all the possible words $A$ that can be contained in any word $Q_{j}$ produced afer $j$ rounds on some initial word $A_{0}$. I.e., if $A$ is produced by the table method, it is possible for $T$, when started with the proper initial word, to produce a word of the form $X A Y$, with $X, Y \in \Sigma^{*}$.

The table method is an iterative method. During the $n$-th iteration step of the table method, first $v$ different words $S_{n, j v+s}, s \in\{0, \ldots, v-1\}$ are produced from each word $S_{n-1, j} 0 \leq j<p_{n-1}$ produced in the previous iteration step $n-1$ of the table method. If $n-1=0$ then the words $S_{0,0}, S_{0,1}, \ldots, S_{0, p_{0}-1}$ are some fixed set of initial words $\in \Sigma^{*}$, usually the appendants.

Every one of the $v$ words $S_{n, j v+s}$ produced from $S_{n-1, j}$ is the word that results after one $s$-round of $T$ on $S_{n-1, j}$. For each of the $v p_{n-1}$ words thus produced, if $S_{n, j v+s}$ is equal to $\epsilon$ or to one of the $S_{x, y}, y<$ $j v+s, x \leq n$ it is marked. If all the $S_{n, j v+s}$ are marked the method halts. If not, then all $S_{n, j v+s}$ that have been marked are removed, the $p_{n}$ remaining words are renumbered as $S_{n, 0,}, S_{n, 1}, S_{n, 2}, \ldots, S_{n, p_{n}-1}$ and the next iteration can be started.

The method is called the table method because the results from the method can often best be represented through tables. To explain the table method and its representation we will apply it to the example of the 3-tag system mentioned in Sec. 1.1 with $w_{0}=00, w_{1}=1101$, setting $S_{0,0}=w_{0}, S_{0,1}=w_{1}$. The following Table shows the first 3 steps of the table method:

\section{hgkjhgkgku}

\section{$p_{1,3}$}

\begin{tabular}{|c|c|c|c|}
\hline jhgkjhg & $\mathrm{j} ; \mathrm{jkh}$ & kjhkjh & jhkjh \\
\hline jhgkjhg & $\mathrm{j} ; \mathrm{jkh}$ & kjhkjh & jhkjh \\
jhgkjhg & $\mathrm{j} ; \mathrm{jkh}$ & kjhkjh & jhkjh \\
\hline \hline
\end{tabular}

\begin{tabular}{|c|c|c||c||c|c|c||c|}
\hline & $w_{0}$ & $w_{1}$ & $w_{1} w_{1}$ & $w_{1} w_{1} w_{0}$ & $w_{1} w_{1} w_{1}$ & $w_{0} w_{1}$ & $\ldots$ \\
\hline$S_{0}$ & $w_{0} \checkmark$ & $w_{1} w_{1}$ & $w_{1} w_{1} w_{0}$ & $w_{1} w_{1} w_{0} w_{0}$ & $w_{1} w_{1} w_{0} w_{1}$ & $w_{0} w_{1} \checkmark$ & $\ldots$ \\
$S_{1}$ & $w_{0} \checkmark$ & $w_{1} \checkmark$ & $w_{1} w_{1} w_{1}$ & $w_{1} w_{1} w_{1} \checkmark$ & $w_{1} w_{1} w_{1} w_{0}$ & $w_{0} w_{0}$ & $\ldots$ \\
$S_{2}$ & $\epsilon \checkmark$ & $w_{0} \checkmark$ & $w_{0} w_{1}$ & $w_{0} w_{1} w_{0}$ & $w_{0} w_{1} w_{1} w_{1}$ & $w_{1} w_{1} \checkmark$ & $\ldots$ \\
\hline
\end{tabular}


The table is read as follows. Each pair of iterative steps of the table method is separated in the table by a double vertical line. The row headed with $S_{x}$ gives the word produced at iteration step $n$ after one $x$-round on the word $S_{n-1, y}$ at the top cell of a column. I.e., the word produced at step $n$ from a given word $S_{n-1, y}$ where the first symbol read in $S_{n-1, y}$ is the $x+1^{\text {th }}$ symbol from the left (i.e. the leftmost $x$ symbols in $S_{n-1, y}$ are deleted without being read).

Columns 2 and 3 give the result for step 1. Since there is only one word left unmarked at step 1, i.e., $w_{1} w_{1}$, we need only one column, column 4 , for step 2 . For step 2 all $v p_{1}=v$ words produced are left unmarked. As a result we need 3 columns for step 3. Now, out of the $v p_{2}=9$ words produced, six are left unmarked. This table allows us to study this example in more detail and it seems that, for this specific example, there will always be words left unmarked.

So why is this method such a useful tool? If we apply the table method to the set of appendants $w_{0}, w_{1}, \ldots, w_{\mu-1}$ of some tag system $T$ (setting each of the $S_{0, i}=w_{i}$ ) this method implies that, if it halts at a given iteration step $n$, then $T$ has a decidable reachability problem. This follows from Lemma 1:

Lemma 1. For any tag system $T$ with deletion number $v$, alphabet $\Sigma$ and appendants $w_{0}, w_{1}, \ldots, w_{\mu-1}$, if the table method halts after a finite number of steps $n$ when applied to $T$ with each $S_{0, i}=w_{i}, 0 \leq i<\mu$ then $T$ will always halt or become periodic on any initial word $A_{0} \in \Sigma^{*}$.

\section{Proof:}

Let $T$ be a $v$-tag system with alphabet $\Sigma$ and corresponding appendants $w_{0}, w_{1}, \ldots, w_{\mu-1}$ for which the table method halts after a finite number of steps $n$ when applied to $T$, with each $S_{0, i}=w_{i}, 0 \leq i<\mu$ and let the union of the appendants and the set of all the different words $S_{i, j}$ that have been produced by the table method after $m$ steps with $0 \leq i \leq m, 0 \leq j<p_{i}$, including $\epsilon$, be denoted as $\mathbb{S}_{m}$. It immediately follows that if the table method halts after $n$ iteration steps, $\mathbb{S}_{n}$ is the finite set of all the possible words that can be produced from the appendants of $T$ by the table method.

Given an initial word $A_{0} \in \Sigma^{*}$, then, after one round of $T$ on $A_{0} T$ produces the word:

$$
Q_{1}=X_{0} S_{0, i_{1}} S_{0, i_{2}} \ldots S_{0, i_{\left\lceil l_{A_{0}} / v\right\urcorner-1}}
$$

with $X_{0}$ one of the appendants $w_{i}$ without its first $\overline{\left(l_{A_{0}} \bmod v\right)}$ letters, each $S_{0, i_{m}} \in \mathbb{S}_{0}=\left\{w_{0}, w_{1}, \ldots, w_{\mu-1}\right\}$. After one more round of $T$ on $Q_{1} T$ produces the word:

$$
Q_{2}=X_{1} S_{1, i_{1}} S_{1, i_{2}} \ldots S_{1, i_{\left\lceil l_{A_{0}} / v\right\rceil-1}}
$$

with each $S_{1, i_{m}} \in \mathbb{S}_{1}$ and $X_{1}$ is one of the $S_{i, j} \in \mathbb{S}_{1}$ minus its first $\overline{\left(l_{Q_{1}} \bmod v\right)}$ letters. Note that the total number of words $S_{1, i_{m}}$ in $Q_{2}$ is equal to $\left\lceil l_{A_{0}} / v\right\rceil-1$. The reason for this is that each $S_{0, i_{m}}$ in $Q_{1}$ produces exactly one word $S_{1, i_{m}}$ in $Q_{2}$, i.e., the word that is produced after one $s$-round on $S_{0, i_{m}}$ where $s$ is determined by the additive complement of the length of the subword preceding $S_{0, i_{m}}$ in $Q_{1}$. Generally speaking, it easily follows from the table method that after $p+1$ rounds of $T$ on $A_{0} T$ produces the word:

$$
Q_{p+1}=X_{p} S_{p, i_{1}} S_{p, i_{2} \ldots} S_{p, i_{\left\lceil l_{A_{0}} / v\right\rceil-1}}
$$

with each $S_{p, i_{m}} \in \mathbb{S}_{p}$ and $X_{p}$ is one of the words $S_{i, j} \in \mathbb{S}_{p}$ minus its first $\overline{\left(l_{Q_{p}} \bmod v\right)}$ letters. Note that the total number of words $S_{p, i_{m}}$ in $Q_{p+1}$ has remained constant. 
Now let us assume that $p=n$. Since we assumed that the table method halts after $n$ steps it follows for any word:

$$
Q_{p+k+1}=X_{p+k} S_{p+k, i_{1}} S_{p+k, i_{2}} \ldots S_{p+k, i_{\left\lceil l_{A_{0}} / v\right\rceil-1}}
$$

produced after $p+k+1$ rounds of $T$ on $A_{0}$, with $k \geq 0$ that each $S_{p+k, i_{m}}$ in $Q_{p+k+1}$ must be one of the words $S_{i, j} \in \mathbb{S}_{p}$ since $\mathbb{S}_{p}=\mathbb{S}_{p+k}$. Similarly, $X_{p+k}$ in $Q_{p+k+1}$, must be one of the $S_{i, j} \in \mathbb{S}_{p}$ minus its first $\overline{\left(l_{Q_{p+k}} \bmod v\right)}$ letters. It now easily follows that $T$ must either halt or become periodic on $A_{0}$. This is the case because the length of any one of the subwords $S_{p+k, i_{m}}$ and the subword $X_{p+k}$ in any word $Q_{p+k+1}$ produced after $p+k+1$ rounds of $T$ on $A_{0}, k \in \mathbb{N}$ is bounded by the length of the lengthiest word in $\mathbb{S}_{p}$. Since the length of the possible productions from $A_{0}$ is thus bounded, $T$ must either halt or become periodic on $A_{0}$.

The proof of Lemma 1 reveals a clear connection between the actual productions of a tag system $T$ and the productions of the table method. We also have the following immediate corollary from this proof:

Corollary 1. Given $v$-tag system $T$ with $\mu$ symbols, appendants $w_{0}, \ldots, w_{\mu-1}, A_{0} \in \Sigma^{*}$ and $\mathbb{S}_{i}, i \in \mathbb{N}$ the union of the appendants and all the different words that have been produced after $i$ iteration steps of the table method applied to the appendants $w_{0}, \ldots, w_{\mu-1}$, then for any word $Q_{j}, j \geq i+1$ produced after $j$ rounds on $A_{0}$ :

$$
Q_{j}=X_{j} V_{j}
$$

where $X_{j}$ is either one of the words in $\mathbb{S}_{i}$ minus its first $\overline{\left(l_{Q_{j-1}} \bmod v\right)}$ letters or $\epsilon$ and $V_{j} \in\left\{\mathbb{S}_{i}\right\}^{*}$

The table method is not only useful if it halts when applied to a given tag system. It can also be used to prove that a tag system will either halt or have unbounded growth, resulting in a non-terminating table. This kind of proof is possible because the method also reveals the "structure" of the possible productions of a given tag system. In general, it should be noted that, although this method is very simple, it is an important instrument to study tag systems.

On the basis of the table method we can now introduce the following definition:

Definition 7. (s-round) We will say that a given tag system $T$ produces a word $\vec{A}_{n}$ after $n s$-rounds of $T$ on $W$, if $\vec{A}_{n}$ is one of the words produced at step $n$ of the table method, with $p_{0}=1, S_{0,0}=W$.

\subsection{General structure of the proof}

In order to prove Theorem 1 only those tag systems with $l_{\min }<2$ and $l_{\max }>2$ need to be taken into account. This follows from Wang's decidability criterion which proves that any tag system $T$ with $l_{\min } \geq v$ or $l_{\max } \leq v$ has a decidable reachability problem. In the remainder, we assume that $l_{\max }=$ $l_{w_{1}}, l_{\text {min }}=l_{w_{0}}$. The symmetrical case is equivalent to this case.

It now follows from Wang's decidability criterion and the fact that $\mu=2$, that we only need to prove Theorem 1 for the following words $w_{0}$ :
I $w_{0}=\epsilon$
II $w_{0}=1$
III $w_{0}=0$ 
These values for $w_{0}$ determine the three global cases of the proof. Each of these three cases will be split into several subcases. These are determined by four different parameters: \#1, the parity ${ }^{2}$ of $l_{w_{1}}, l_{w_{1}}$ and the parities of the number of 0 symbols separating consecutive 1 symbols in $w_{1}$.

\subsubsection{Parameter 1: \#1}

The total number \#1 of 1 symbols in the appendants of a given tag system $T$ is the parameter used for determining the main subcases for each of the three global cases. For each of these three global cases $\mathbf{I}$, II and III treshold values $n_{\mathbf{I}}, n_{\mathbf{I I}}$ and $n_{\text {III }}$ for $\# 1$ are determined. It is these treshold values that allow us to reduce the infinite number of subcases for each of the main cases to a finite number. It will be proven that the infinite number of tag systems covered by the three main cases for which $\# 1 \geq n_{\mathbf{I}}$ (for Case I), $\# 1 \geq n_{\text {II }}$ (for Case II) and \#1 $\geq n_{\text {III }}$ (for Case III) always have unbounded growth (except, possibly, for a determined set of initial words), while the finite number of tag systems determined by the three gobal cases for which $\# 1<n_{\mathbf{I}}$ (for Case I), $\# 1<n_{\text {II }}$ (for Case II) and \#1< $n_{\text {III }}$ (for Case III) always halt or become periodic (except, possibly, for a determined class of initial words).

The parameter \#1 is varied going from 0 up to its treshold value. Capital letters A, B, C,... will be used to enumerate these subcases.

One of the main reasons for the significance of \#1 is that for any tag system $T$ the symbol 1 corresponds with $w_{1}$, the longest appendant and the only possibility for increasing the length of a given word. Furthermore, \#1 also has an impact on the length of $w_{1}$.

\subsubsection{Parameter 2: The parity of $l_{w_{1}}$}

The parity of $l_{w_{1}}$ plays a major role in Case $\mathbf{I}$ and is used as a parameter to further split-up each of the main subcases of Case I determined by \#1. The parameter is, however, not used in the further factorization of the subcases for cases II and III.

The reason that $l_{w_{1}}$ only plays a significant role for Case $\mathbf{I}$ is the fact that $w_{0}=\epsilon$. This means that any word $Q_{j}$ produced after $j$ rounds on some initial word is always either equal to $w_{1}^{n}$ or $w_{1}^{-} w_{1}^{n}$ with $n \in \mathbb{N}$. I.e., every word $Q_{j}$ is a word consisting entirely of words $w_{1}$.

\subsubsection{Parameter 3: $l_{w_{1}}$}

The next parameter $l_{w_{1}}$ is only significant with respect to Case II where it is used as a parameter to further split-up the subcases determined by \#1. For tag systems $T$ with $w_{0}=1$ (Case II), the "second-order effect" of reading a 0 can be an increase in the length of a word. Indeed, since for any such $T$ a 0 symbol produces a 1 symbol and a 1 symbol produces $w_{1}$ the effect of reading a 0 symbol can indirectly result in an increase of the length of a given word. As a consequence, we not only need to determine a treshold value with respect to $\# 1$ but also with respect to $l_{w_{1}}$ for Case II. This is not necessary for cases $\mathbf{I}$ and III. For Case I, the effect of reading a 0 is the production of $\epsilon$ so it always results in a decrease of the length of a given word. For Case III, the effect of reading a 0 in a given word $Q_{j}$ is also a decrease of the length of $Q_{j}$ since two letters are deleted and only one, i.e., 0 , is appended.

\footnotetext{
${ }^{2}$ The parity of a number $x$ is the property of it being even or odd.
} 


\subsubsection{Parameter 4: The parity of the number of 0 symbols separating consecutive 1 symbols in $w_{1}$}

The parity of the number of 0 symbols separating consecutive 1 symbols in $w_{1}$ plays a significant role in each of the three global cases I, II and III. It is the parameter used to further split-up the subcases already determined by the other parameters that are relevant for a given case. (For Case $\mathbf{I}$ this is the first and the second parameter, for Case II the first and the third, and for Case III only the first).

The significance of the parity of the number of 0 symbols separating consecutive 1 symbols in $w_{1}$ has to do with the fact that, since we are dealing with 2-tag systems, an even number of 0 symbols separating two 1 symbols implies that always one of the two will be read by the tag system, while an odd number implies that either both 1 symbols or none of the two 1 symbols will be read in $w_{1}$. Clearly, this parameter can only start to play a role if the number of 1 symbols in $w_{1} \geq 2$.

We will now turn to the details of the proof of Theorem 1.

\subsection{Proof of Theorem 1}

Case I. $\mathbf{w}_{0}=\epsilon$.

As explained in Sec. 2.2, the parameters used to prove this case are $\# 1$, the parity of $l_{w_{1}}$ and the different parities of the number of 0 symbols between consecutive 1 symbols in $w_{1}$. The parity of $l_{w_{1}}$ only starts to play a role when $\# 1>0$ (starting from Case I.B). The parities of the number of 0 symbols between consecutive 1 symbols in $w_{1}$ is only relevant once $\# 1 \geq 2$ (starting from Case I.C).

Case I.A. \#1 $=0\left(w_{0}=\epsilon\right)$.

If $\# 1=0$ and $w_{0}=\epsilon$ then $w_{1}=0^{l_{w_{1}}}$. This immediately implies that any tag system $T$ from this class must always halt, irrespective of the length of $w_{1}$. The reason for this is that whatever the initial word $A_{0}$ the word $Q_{1}$ produced after one round of $T$ on $A_{0}$ consists entirely of 0 symbols. We then immediately have that the word $Q_{2}$ produced after one more round on $Q_{1}$ is equal to $\epsilon$ since $w_{0}=\epsilon$.

Case I.B. $\# 1=1\left(w_{0}=\epsilon\right)$.

We split this case into two subcases determined by the parity of $l_{w_{1}}$.

Case I.B.1. $l_{w_{1}} \equiv 0 \bmod 2\left(\# 1=1, w_{0}=\epsilon\right)$

Since $\# 1=1$ and $l_{w_{1}} \equiv 0 \bmod 2$, either $w_{1}=0^{r_{1}} 10^{\ddot{t_{1}}}$ or $w_{1}=0^{\ddot{r}_{1}} 10^{\dot{t_{1}}}$. Table 2 proves that the table method halts for any tag system $T$ from this class with $w_{1}=0^{\dot{r}_{1}} 10^{\ddot{t_{1}}}$.

Table 2: $w_{1}=0^{\dot{r_{1}}} 10^{\ddot{t_{1}}}$

\begin{tabular}{|l|l|l|}
\hline & $w_{0}$ & $w_{1}$ \\
\hline$S_{0}$ & $\epsilon$ & $\epsilon$ \\
$S_{1}$ & $\epsilon$ & $w_{1} \checkmark$ \\
\hline
\end{tabular}

Since the table method halts, $T$ will always halt or become periodic on any initial word $A_{0}$. The case 
with $w_{1}=0^{\ddot{r_{1}}} 10^{\dot{t_{1}}}$ is symmetrical to this case.

Case I.B.2. $l_{w_{1}} \equiv 1 \bmod 2\left(\# 1=1, w_{0}=\epsilon\right)$.

Since $\# 1=1$ and $l_{w_{1}} \equiv 1 \bmod 2$, either $w_{1}=0^{r_{1}} 10^{\dot{t_{1}}}$ or $w_{1}=0^{\ddot{r}} 10^{\ddot{t_{1}}}$. Table 3 proves the case for $w_{1}=0^{r_{1}} 10^{\dot{t_{1}}}$.

Table 3: $w_{1}=0^{r_{1}} 10^{\dot{t_{1}}}$

\begin{tabular}{|l|l|l|}
\hline & $w_{0}$ & $w_{1}$ \\
\hline$S_{0}$ & $\epsilon$ & $\epsilon \checkmark$ \\
$S_{1}$ & $\epsilon$ & $w_{1} \checkmark$ \\
\hline
\end{tabular}

The table method halts at step 1 , all words produced from $w_{0}$ and $w_{1}$ being marked. It thus follows that any tag system $T$ from this class with $w_{1}=0^{r_{1}} 10^{t_{1}}$ will always either halt or become periodic on some initial word $A_{0}$.

The case with $w_{1}=0^{\ddot{r_{1}}} 10^{\ddot{t_{1}}}$ is symmetrical to this case.

Case I.C. $\# 1=2\left(w_{0}=\epsilon\right)$.

We split the case into two subcases determined by the parity of $l_{w_{1}}$. Note that from now on, \#1>1. Since $w_{0}=\epsilon$, this means that parameter 4 from Sec. 2.2, the parity of the number of 0 symbols separating consecutive 1 symbols in $w_{1}$, starts to play its role.

Case I.C.1. $l_{w_{1}} \equiv 0 \bmod 2\left(\# 1=2, w_{0}=\epsilon\right)$.

We split the case into two subcases determined by the parity of the number of 0 symbols between the two 1 symbols in $w_{1}$. I.e., a case with $w_{1}=0^{\ddot{r_{1}}} 10^{\ddot{x_{1}}} 10^{\ddot{t_{1}}}$ (or, equivalently, $w_{1}=0^{\dot{r}_{1}} 10^{\ddot{x_{1}}} 10^{\dot{t_{1}}}$ ) and one with $w_{1}=0^{\dot{r_{1}}} 10^{\dot{x_{1}}} 10^{\ddot{t_{1}}}$ (or, equivalently, $w_{1}=0^{\ddot{r_{1}}} 10^{\dot{x_{1}}} 10^{\dot{t_{1}}}$ ).

Case I.C.1.a. $w_{1}=0^{\ddot{r_{1}}} 10^{\ddot{x_{1}}} 10^{\ddot{t_{1}}}$ or $w_{1}=0^{\dot{r_{1}}} 10^{\ddot{x_{1}}} 10^{\dot{t_{1}}}\left(\# 1=2, l_{w_{1}} \equiv 0 \bmod 2, w_{0}=\epsilon\right)$. Let $w_{1}=0^{\ddot{r} 1} 10^{\ddot{x_{1}}} 10^{\ddot{t_{1}}}$. The case is proven by Table 4 .

Table 4: $w_{1}=0^{\ddot{r_{1}}} 10^{\ddot{x_{1}}} 10^{\ddot{t_{1}}}$

\begin{tabular}{|l|l|l|}
\hline & $w_{0}$ & $w_{1}$ \\
\hline$S_{0}$ & $\epsilon$ & $w_{1} \checkmark$ \\
$S_{1}$ & $\epsilon$ & $w_{1} \checkmark$ \\
\hline
\end{tabular}

Since the table method always halts for this case, it follows that any tag system $T$ from this class will always either halt or become periodic. The case with $w_{1}=0^{\dot{r}_{1}} 10^{\ddot{x_{1}}} 10^{\dot{t}_{1}}$ reduces to this case, since the 
table method results in the same productions.

Case I.C.1.b. $w_{1}=0^{\dot{r_{1}}} 10^{\dot{x_{1}}} 10^{\ddot{t_{1}}}$ or $w_{1}=0^{\ddot{r_{1}}} 10^{\dot{x_{1}}} 10^{\dot{t_{1}}}\left(\# 1=2, l_{w_{1}} \equiv 0 \bmod 2, w_{0}=\epsilon\right)$. Let $w_{1}=0^{\dot{r}_{1}} 10^{\dot{x_{1}}} 10^{\ddot{t_{1}}}$. Table 5 proves this case.

Table 5: $w_{1}=0^{r_{1}} 10^{\dot{x_{1}}} 10^{\ddot{t_{1}}}$

\begin{tabular}{|l|l|l||l||l||l||}
\hline & $w_{0}$ & $w_{1}$ & $w_{1} w_{1}$ & $\ldots$ & $\left(w_{1} w_{1}\right)^{n}$ \\
\hline$S_{0}$ & $\epsilon$ & $\epsilon$ & $\epsilon$ & $\ldots$ & $\epsilon$ \\
$S_{1}$ & $\epsilon$ & $w_{1} w_{1}$ & $w_{1} w_{1} w_{1} w_{1}$ & $\ldots$. & $\left(w_{1} w_{1}\right)^{2 n}$ \\
\hline
\end{tabular}

It easily follows from Table 5 that any tag system $T$ from this case will either halt or have unbounded growth. So, given some initial word $A_{0}$, which of the two kinds of behavior will the tag systems have? Let $\vec{B}_{0}=\epsilon, \vec{B}_{1}=w_{1}^{2}$. Clearly, if $Q_{j}$, the word produced after $j$ rounds on $A_{0}$, is a word of the form $w_{1}^{n}$, then $Q_{j+1}=\vec{B}_{0}^{n}=\epsilon$ because $l_{w_{1}}$ is even. Similarly, if $Q_{j}$ is a word of the form $w_{1}^{-} w_{1}^{n}$ then $Q_{j+1}=\vec{B}_{1}^{-} \vec{B}_{1}^{n}=w_{1}^{-} w_{1}^{2 n}$. From this it immediately follows that if $l_{A_{0}}$ is even that $T$ will halt after at most two rounds on $A_{0}$. If $l_{A_{0}}$ is odd then $Q_{1}=w_{1}^{-} w_{1}^{n}$ and thus it easily follows that $T$ has unbounded growth on $A_{0}$.

The case with $w_{1}=0^{\ddot{r_{1}}} 10^{\dot{x_{1}}} 10^{\dot{t}_{1}}$ is symmetrical to this case.

Case I.C.2. $l_{w_{1}} \equiv 1 \bmod 2\left(\# 1=2, w_{0}=\epsilon\right)$.

We need to split the case into two subcases determined by the parity of the number of 0 symbols separating the two consecutive 1 symbols in $w_{1}$. I.e., a case with $w_{1}=0^{\ddot{r}_{1}} 10^{\dot{x_{1}}} 10^{\ddot{t}_{1}}$ (or, equivalently, $w_{1}=0^{r_{1}} 10^{\dot{x_{1}}} 10^{\dot{t_{1}}}$ ) and a case with $w_{1}=0^{\dot{r_{1}}} 10^{\ddot{x_{1}}} 10^{\ddot{t_{1}}}$ (or equivalently $w_{1}=0^{\ddot{r}_{1}} 10^{\ddot{x_{1}}} 10^{\dot{t_{1}}}$ ).

Case I.C.2.a. $w_{1}=0^{r_{1}} 10^{\ddot{x_{1}}} 10^{\ddot{t_{1}}}$ or $w_{1}=0^{\ddot{r_{1}}} 10^{\ddot{x_{1}}} 10^{\dot{t_{1}}}\left(\# 1=2, l_{w_{1}} \equiv 1 \bmod 2, w_{0}=\epsilon\right)$.

Let $w_{1}=0^{r_{1}} 10^{\ddot{x_{1}}} 10^{\ddot{t_{1}}}$. Table 6 proves that the table method always halts if $w_{1}=0^{r_{1}} 10^{\ddot{x_{1}}} 10^{\ddot{t_{1}}}$ and thus that for this case $T$ will always halt or become perodic.

Table 6: $w_{1}=0^{\dot{r_{1}}} 10^{\ddot{x_{1}}} 10^{\ddot{t_{1}}}$

\begin{tabular}{|l|l|l|}
\hline & $w_{0}$ & $w_{1}$ \\
\hline$S_{0}$ & $\epsilon$ & $w_{1} \checkmark$ \\
$S_{1}$ & $\epsilon$ & $w_{1} \checkmark$ \\
\hline
\end{tabular}

The case with $w_{1}=0^{\ddot{r_{1}}} 10^{\ddot{x_{1}}} 10^{\dot{t_{1}}}$ reduces to this case since the tables resulting from the table method are, for both cases, identical. 
Case I.C.2.b. $w_{1}=0^{\ddot{r_{1}}} 10^{\dot{x_{1}}} 10^{\ddot{t_{1}}}$ or $w_{1}=0^{\dot{r_{1}}} 10^{\dot{x_{1}}} 10^{\dot{t_{1}}}\left(\# 1=2, l_{w_{1}} \equiv 1 \bmod 2, w_{0}=\epsilon\right)$. Let $w_{1}=0^{\ddot{r_{1}}} 10^{\dot{x}_{1}} 10^{\ddot{t_{1}}}$. Table 7 proves that the table method always halts if $w_{1}=0^{\ddot{r}_{1}} 10^{\dot{x_{1}}} 10^{\ddot{t_{1}}}$ and thus that for this case $T$ will always halt or become perodic.

Table 7: $w_{1}=0^{\ddot{r_{1}}} 10^{\dot{x_{1}}} 10^{\ddot{t_{1}}}$

\begin{tabular}{|l|l|l||l|}
\hline & $w_{0}$ & $w_{1}$ & $w_{1} w_{1}$ \\
\hline$S_{0}$ & $\epsilon$ & $w_{1} w_{1}$ & $w_{1} w_{1} \checkmark$ \\
$S_{1}$ & $\epsilon$ & $\epsilon \checkmark$ & $w_{1} w_{1} \checkmark$ \\
\hline
\end{tabular}

The case with $w_{1}=0^{\dot{r_{1}}} 10^{\dot{x_{1}}} 10^{\dot{t_{1}}}$ is symmetrical to this case. The only difference is that the results from Table 7 for shifts $S_{0}$ and $S_{1}$ need to be switched.

Case I.D $\# 1 \geq 3\left(w_{0}=\epsilon\right)$.

This case needs to be split in two subcases determined by the parity of $l_{w_{1}}$.

Case I.D.1 $l_{w_{1}} \equiv 0 \bmod 2\left(\# 1 \geq 3, w_{0}=\epsilon\right)$.

We split the case into two subcases determined by the parity of the number of 0 symbols between consecutive 1 symbols in $w_{1}$, i.e., a case with $w_{1}=0^{r_{1}} 10^{\dot{x}_{1}} 10^{\dot{x}_{2}} 10^{\dot{x_{3}}} \ldots 0^{\dot{x}_{\# 1-1}} 10^{t_{1}}$, with any $\dot{x}_{i}, 0<i<\# 1$ odd and a case with $w_{1}=0^{r_{1}} 10^{x_{1}} 10^{x_{2}} 10^{x_{3}} \ldots 0^{x_{\# 1-1}} 10^{t_{1}}$, with at least one $x_{i}, 0<i<\# 1$ that is even.

Case I.D.1.a $w_{1}=0^{r_{1}} 10^{x_{1}} 10^{x_{2}} 10^{x_{3}} \ldots 0^{x_{\# 1-1}} 10^{t_{1}}$, at least one $x_{i}$ even $\left(w_{0}=\epsilon, \# 1 \geq 3, l_{w_{1}} \equiv\right.$ $0 \bmod 2$ ).

Note that for any tag system $T$ from this class either at least two 1 symbols or at least one 1 symbol is read after one $s$-round on $w_{1}$ since $w_{1}$ contains at least two consecutive 1 symbols that are separated by an even number of 0 symbols.

The ultimate behaviour of any tag system $T$ from this class can be easily determined. Given $w_{1}$ one first needs to determine how many 1 symbols will be read by $T$ in $w_{1}$ when entered with shift $S_{0}$ and $S_{1}$ respectively. Let $b_{0}$ be the number of 1 symbols read when $w_{1}$ is entered with shift $S_{0}$ and $b_{1}$ the number of 1 symbols read when $w_{1}$ is entered with shift $S_{1}$ and let $B_{0}$ be the word produced after one 0 -round on $w_{1}$ and $B_{1}$ the word produced after one 1-round on $w_{1}$. Then, since $l_{w_{1}} \equiv 0 \bmod 2$, and $w_{0}=\epsilon$, for any word $Q_{j}$ produced after $j>1$ rounds on some initial word $A_{0}$ we have that $Q_{j}=B_{0}^{m}=\left(w_{1}\right)^{m b_{0}}$ and $Q_{j+1}=B_{0}^{m b_{0}}$ (if $l_{A_{0}}$ is even) or $B_{1}^{-} B_{1}^{m}=\left(w_{1}^{b_{1}}\right)^{-} w_{1}^{m b_{1}}$ and $Q_{j+1}=\left(B_{1}^{b_{1}}\right)^{-} B_{1}^{m b_{1}}$ (if $l_{A_{0}}$ is odd). This is the reason why the ultimate behavior of some of the tag system $T$ covered by this case depends on the parity of $l_{A_{0}}$. There are two possibilities:

1. $b_{0} \geq 2, b_{1}=1$ (or vice versa). If at least one 1 is read in the initial word, $T$ will either have unbounded growth or become periodic depending on the parity of the initial word. Else $T$ will halt.

2. $b_{0} \geq 2, b_{1} \geq 2$ (or vice versa). $T$ will always have unbounded growth, whatever the parity of the initial word if at least one 1 is read in the initial word. Else $T$ will halt. 
Case I.D.1.b $w_{1}=0^{r_{1}} 10^{\dot{x_{1}}} 10^{\dot{x_{2}}} 10^{\dot{x_{3}}} \ldots 0^{\dot{x_{\# 1-1}}} 10^{t_{1}}$, with any $\dot{x}_{i}, 0<i<\# 1$, odd $\left(\# 1 \geq 3, l_{w_{1}} \equiv\right.$ $\left.0 \bmod 2, w_{0}=\epsilon\right)$

Note that since all 1 symbols in $w_{1}$ are separated by an odd number of 0 symbols it immediately follows that after one $s$-round on $w_{1} T$ produces either $\epsilon$ or $w_{1}^{\# 1}$. From this it immediately follows that $T$ either halts or has unbounded growth on any word $A_{0}$ depending on the parity of $A_{0}$ (see Case I.C.1.b. for more details).

Case I.D.2. $\# 1 \geq 3, l_{w_{1}} \equiv 1 \bmod 2\left(w_{0}=\epsilon\right)$.

The case is split into two subcases determined by the parity of the number of 0 symbols between consecutive 1 symbols in $w_{1}$, i.e., a case with $w_{1}=0^{r_{1}} 10^{\dot{x_{1}}} 10^{\dot{x_{2}}} 10^{\dot{x_{3}}} \ldots 0^{\dot{x}_{\# 1-1}} 10^{t_{1}}$, with every $\dot{x}_{i}, 0<i<\# 1$ odd and a case with $w_{1}=0^{r_{1}} 10^{x_{1}} 10^{x_{2}} 10^{x_{3}} \ldots 0^{x_{\# 1-1}} 10^{t_{1}}$ for which there is at least one $x_{i}, 0<i<\# 1$, that is even.

Case I.D.2.a $w_{1}=0^{r_{1}} 10^{x_{1}} 10^{x_{2}} 10^{x_{3}} \ldots 0^{x_{\# 1-1}} 10^{t_{1}}$, at least one $x_{i}$ even $\left(w_{0}=\epsilon, \# 1 \geq 3, l_{w_{1}} \equiv\right.$ $1 \bmod 2$ )

Note that any tag system $T$ from this class will either read at least two 1 symbols or one 1 symbol in $w_{1}$, depending on the shift with which $w_{1}$ is entered. This immediately implies that $T$ can never halt if at least one 1 is read in the initial word because the number of $w_{1}$ words either increases or stays the same following each round. Now, since $l_{w_{1}}$ is odd, it follows that after one $s$-round on $w_{1}^{2}, T$ produces $w_{1}^{n}, n \geq 3$. The result of unbounded growth thus immediately follows if the word $Q_{1}$ produced after one round on $A_{0}$ is either $w_{1}^{n}, n \geq 2$ or $w_{1}^{-} w_{1}^{n}, n \geq 1$. This is always the case if at least two 1 symbols are read in $A_{0}$. Otherwise, if $Q_{1}=w_{1}$ or $Q_{1}=w_{1}^{-}$(only one 1 symbol is read in $A_{0}$ ) then it can be easily checked that $Q_{3}=w_{1}^{m}, m \geq 2$ or $Q_{3}=w_{1}^{-} w_{1}^{m}, m \geq 1$ and thus we again have unbounded growth. This means that $T$ will always have unbounded growth on any word $A_{0}$ if $T$ reads at least one 1 symbol in $A_{0}$.

Case I.D.2.b $w_{1}=0^{r_{1}} 10^{\dot{x_{1}}} 10^{\dot{x_{2}}} 10^{\dot{x_{3}}} \ldots 0^{\dot{x}_{\# 1-1}} 10^{t_{1}}\left(\# 1 \geq 3, l_{w_{1}} \equiv 1 \bmod 2, w_{0}=\epsilon\right)$.

Note that since all 1 symbols in $w_{1}$ are separated by an odd number of 0 symbols we always have that $T$ produces either $w_{1}^{\# 1}$ or $\epsilon$ after one $s$-round on $w_{1}$. Since $l_{w_{1}}$ is odd every second word $w_{1}$ is entered with a different shift, and this implies that every pair of words $w_{1}$ results in the production of $w_{1}^{\# 1}$. Since $\# 1 \geq 3$ it follows easily that any tag system $T$ from this class will always have unbounded growth on any initial word $A_{0}$ if the word $Q_{1}=w_{1}^{m}, m \geq 2$ or $Q_{1}=w_{1}^{-} w_{1}^{m}, m \geq 1$. It is also easily checked that $T$ will have unbounded growth on $A_{0}$ if $Q_{1}=w_{1}$ or $Q_{1}=w_{1}^{-}$and $Q_{2}=w_{1}^{\# 1}$ or $Q_{2}=w_{1}^{-} w_{1}^{\# 1-1}$. In all other cases, $T$ halts on $A_{0}$.

Case II. $w_{0}=1$.

As explained in Sec. 2.2, unlike cases I and III, $l_{w_{1}}$ is a parameter that needs to be reckoned with for this case. Also \#1 and the different parities of the number of 0 symbols between consecutive 1 symbols in $w_{1}$ are used in the proof of this case.

Each subcase defined by $\# 1$, will be factorized according to increasing values for $l_{w_{1}}$ up until a certain treshold value. Since $w_{0}=1$, the parities of the number of 0 symbols between consecutive 1 symbols in $w_{1}$ only start to play a role once \#1 $>2$ (starting from Case II.C).

Note that the smallest value for $l_{w_{1}}$ is always equal to 3 for each subcase defined by $\# 1$. The reason for 
this is that we only need to take into account those cases with $l_{w_{1}} \geq 3$ because of Wang's decidability criterion which states that any tag systems with $l_{\max } \leq v$ has a decidable reachability problem.

It is trivial to prove that any tag system with $w_{0}=1$ (Case II) always halts on $A_{0}=0$. In what follows we will thus only consider initial words $A_{0} \neq 0$.

The following Lemma is an important tool for the proofs of cases II.A.3 and II.B.2-6. The reason for this is that for each of these cases it can be easily proven that any word $Q_{j}$ produced after $j$ rounds on the initial word $A_{0}$ is composed of subwords from a given set such that for any such subword $T$ always produces a word from that same set in $Q_{j+1}$.

Lemma 2. Given a $v$-tag system $T$ with alphabet $\Sigma$ and corresponding appendants $w_{0}, w_{1}, \ldots, w_{\mu-1}$, some initial word $A_{0} \in \Sigma^{*}$ and $\mathbb{W}=\left\{W_{1}, W_{1} \ldots, W_{m}\right\}$ some set of words $\in \Sigma^{*}$. If one can prove that there is an $n \in \mathbb{N}$ and a set $\mathbb{W}^{\prime} \subseteq \mathbb{W}$ such that for any word:

$$
Q_{j}=X_{j} V_{j} \quad X_{j} \in\left\{\epsilon, W_{1}^{-}, W_{2}^{-}, \ldots, W_{m}^{-}\right\}, V_{j} \in \mathbb{W}^{*}
$$

that contains at least $p$ words from the set $\mathbb{W}^{\prime}$ the following holds (a) there is always at least one subword in $Q_{j}$ from the set $\mathbb{W}$ from which $T$ produces $W_{i} W_{i^{\prime}}$ in $Q_{j+n}$ with $W_{i}, W_{i^{\prime}} \in \mathbb{W}$ (b) for any word $W_{k} \in \mathbb{W}, W_{i} W_{i^{\prime}} \neq W_{k}$, (c) for every other subword in $Q_{j}$ from the set $\mathbb{W} T$ produces at least one word from that same set in $Q_{j+n}$, and (d) there are at least $p$ words from the set $\mathbb{W}^{\prime}$ in $Q_{j+n}$, then $T$ has unbounded growth on any word $A_{0}$ from which $T$ produces a word $Q_{j}$ after $j$ rounds.

\section{Proof:}

The proof is trivial

Case II.A. $\# 1=1\left(w_{0}=1\right)$.

The case is split into 4 cases, according to the value of $l_{w_{1}}$. Remember that the smallest value for $l_{w_{1}}=3$ because of Wang's decidability criterion.

Case II.A.1. $l_{w_{1}}=3\left(w_{0}=1, \# 1=1\right)$.

Note that since $l_{w_{1}}=3, \# 1=1$ and $w_{0}=1, w_{1}=000$.

The result is proven through Table 8:

Table 8: Case $w_{1}=000$

\begin{tabular}{|l|l|l||l|}
\hline & $w_{0}$ & $w_{1}$ & $w_{0} w_{0}$ \\
\hline$S_{0}$ & $w_{1} \checkmark$ & $w_{0} w_{0}$ & $w_{1} \checkmark$ \\
$S_{1}$ & $\epsilon \checkmark$ & $w_{0} \checkmark$ & $w_{1} \checkmark$ \\
\hline
\end{tabular}

Since the table method halts, this tag system will always either halt or become periodic on any initial word $A_{0}$. 
Case II.A.2. $l_{w_{1}}=4\left(w_{0}=1, \# 1=1\right)$.

Since $l_{w_{1}}=4, \# 1=1$ and $w_{0}=1, w_{1}=0000$. Table 9 proves that the table method always halts for $T$ and thus that $T$ will always either halt or become periodic on any initial word $A_{0}$.

Table 9: $l_{w_{1}}=0000$

\begin{tabular}{|l|l|l||l|}
\hline & $w_{0}$ & $w_{1}$ & $w_{0} w_{0}$ \\
\hline$S_{0}$ & $w_{1}$ & $w_{0} w_{0}$ & $w_{1} \checkmark$ \\
$S_{1}$ & $\epsilon \checkmark$ & $w_{0} w_{0}$ & $w_{1} \checkmark$ \\
\hline
\end{tabular}

Case II.A.3. $l_{w_{1}}=5\left(w_{0}=1, \# 1=1\right)$.

If $l_{w_{1}}=5, \# 1=1, w_{1}=00000$. This tag system always has unbounded growth except for a finite set of initial words on which $T$ either halts or becomes periodic. This can be proven by applying Lemma 2 . First of all, note that after one $s$-round on $w_{1} T$ either produces $w_{0}^{3}$ or $w_{0}^{2}$. From $w_{0}^{2} T$ again produces $w_{1}$, from $w_{0}^{3} T$ produces either $w_{1}^{2}$ or again $w_{1}$. It is the possibility of producing $w_{1}^{2}$ from $w_{1}$ after two $s$-rounds on $w_{1}$ that allows for unbounded growth in this tag system.

It follows from Corollary 1 that for any word $Q_{j}, j \geq 2$ we have that:

$$
Q_{j}=X_{j} V_{j} \quad X_{j} \in\left\{\epsilon, w_{0}, w_{1}^{-}\right\}, V_{j} \in\left\{w_{0}^{2}, w_{0}^{3}, w_{1}\right\}^{*}
$$

Note that $V_{j}$ cannot contain a word $w_{0}$ that is neither preceded nor followed by a word $w_{0}$. The reason for this is that $w_{0}$ can only be produced from $w_{1}$, but then $w_{0}$ must be either preceded or followed by at least one word $w_{0}$.

It now easily follows that after one round of $T$ on $Q_{j}$ every subword in $Q_{j}$ from the set $\left\{w_{0}, w_{1}^{-}, w_{1}, w_{0}^{2}, w_{0}^{3}\right\}$ will again result in at least one subword from that same set in $Q_{j+1}$. Furthermore, if $Q_{j}$ contains either $w_{1}^{2}$ or $w_{1}^{-} w_{1}$ then we can prove that also the other conditions of Lemma 2 are met. I.e., for any such word $Q_{j}$ it can be proven that there is at least one subword in $Q_{j}$ from the set $\left\{w_{1}^{-}, w_{1}, w_{0}^{2}, w_{0}^{3}\right\}$ that results in the production of a new word that consists of at least two words from that same set in $Q_{j+i}, i \in\{2,4,6\}$ and differs from any word in that set (conditions a and b), any other subword in $Q_{j}$ from the set $\left\{w_{0}, w_{1}^{-}, w_{1}, w_{0}^{2}, w_{0}^{3}\right\}$ again results in at least one word from that same set in $Q_{j+i}$ (condition c), $Q_{j+i}$ again contains either $w_{1}^{2}$ or $w_{1}^{-} w_{1}$ (condition d).

It can be easily checked that if $T$ produces the word $Q_{1}$ after one round on $A_{0}$ with $Q_{1}$ one of the following words $w_{0} w_{1} W, w_{1} w_{0} W, w_{1} w_{1} W$, or $w_{0} w_{0} w_{0} W, W \in\left\{w_{0}, w_{1}\right\}^{*}$ then it takes at most four more rounds to produce a word that contains $w_{1}^{2}$ or $w_{1}^{-} w_{1}$. The only initial words that do not result in such words $Q_{1}$ are: $(\mathbf{0}), \mathbf{1}, \mathbf{0} 0,01,10,11,000,010,001,011,0000,0100,0001,0101,00000,01010,00010$ or 01000 . $T$ always becomes periodic on any of these words.

We will now prove that Lemma 2 is indeed applicable for words $Q_{j}$ that contain $w_{1}^{2}$ or $w_{1}^{-} w_{1}$ at least once. Let $Q_{j}$ be such a word. Clearly, from $w_{1}^{2}$ or $w_{1}^{-} w_{1}$ in $Q_{j} T$ produces $w_{0}^{5}$ or $w_{0}^{5^{-}}$in $Q_{j+1}$ and thus either again $w_{1}^{2}$ or $w_{1}^{3}$ in $Q_{j+2}$. If $w_{1}^{3}$ is produced in $Q_{j+2}$ from $w_{1}^{2}$ in $Q_{j}$ then all conditions of lemma 2 are met. Indeed, from $w_{1}^{2}$ or $w_{1}^{-} w_{1}$ in $Q_{j}$ a new word is produced in $Q_{j+2}$ that is a combination of two words from the set $\left\{w_{1}^{-}, w_{1}, w_{0}^{2}, w_{0}^{3}\right\}$ and different from any of the words from that set (conditions a and b), every other subword in $Q_{j}$ from the set $\left\{w_{1}^{-}, w_{1}, w_{0}^{2}, w_{0}^{3}\right\}$ must result in at least one subword from 
that same set (condition c) and $Q_{j+2}$ again contains $w_{1}^{2}$ (condition d).

If $w_{1}^{2}$ reproduces itself in $Q_{j+2}$ then this means that $w_{0}^{5}$ in $Q_{j+1}$ produced from $w_{1}^{2}$ in $Q_{j}$ is entered with shift 1 . We then have that $Q_{j+1}=w_{0}^{4} W_{j+1}$ or $Q_{j+1}=X_{j+1} W_{j+1,1} w_{0}^{5} W_{j+1,2}, W_{j+1}, W_{j+1,1}, W_{j+1,2} \in$ $\left\{w_{0}^{2}, w_{0}^{3}, w_{1}\right\}^{*}$. Note that in this last case $l_{X_{j+1} W_{j+1,1}}$ is odd since else $w_{0}^{5}$ in $Q_{j+1}$ would be entered with shift 0 , thus resulting in the production of $w_{1}^{3}$ instead of $w_{1}^{2}$ in $Q_{j+2}$.

The following list gives all the possible words $Q_{j}$ that contain $w_{1}^{2}$ or $w_{1}^{-} w_{1}$ at least once and from which $T$ produces the word $Q_{j+1}=w_{0}^{4} W_{j+1}$. It proves that all conditions of Lemma 2 are met. I.e. there is an $i(i \in\{2,4,6\})$ such that a subword is produced in $Q_{j+i}$ that is the concatenation of at least two words from the set $\left\{w_{0}, w_{1}^{-}, w_{1}, w_{0}^{2}, w_{0}^{3}\right\}$ and is different from any word from that set (conditions a and b) and $Q_{j+i}$ again contains $w_{1}^{2}$ or $w_{1}^{-} w_{1}$ at least once (condition d). Note that in order to produce a word $Q_{j+1}=w_{0}^{4} W_{j+1}, l_{Q_{j}}$ must be odd.

$$
\begin{aligned}
& Q_{j}=w_{1}^{2} \mathbf{w}_{1} W_{j} \quad Q_{j+1}=w_{0}^{4} w_{0}^{3} W_{j+1} \\
& Q_{j+2}=X_{j+2} w_{1} \mathbf{w}_{1}^{2} W_{j+2}, X_{j+2} \in\left\{w_{1}^{-}, w_{1}\right\} \\
& Q_{j}=w_{1}^{2} \mathbf{w}_{\mathbf{0}}^{\mathbf{3}} W_{j} \quad Q_{j+1}=w_{0}^{4} w_{1}^{2} W_{j+1} \\
& Q_{j}=w_{1}^{2} w_{0}^{2} W_{j} \quad Q_{j+1}=w_{0}^{4} w_{1} W_{j} \\
& Q_{j}=w_{1}^{-} \mathbf{w}_{\mathbf{1}} \\
& Q_{j+4}=w_{1}^{-} \mathbf{w}_{1}^{2} \\
& Q_{j}=w_{1}^{-} w_{1} \mathbf{w}_{\mathbf{1}} \\
& l_{Q_{j}} \equiv 0 \bmod v \\
& Q_{j+2}=X_{j+2} w_{1} \mathbf{w}_{\mathbf{0}}^{\mathbf{5}} W_{j+2}, X_{j+2} \in\left\{w_{1}^{-}, w_{1}\right\} \\
& Q_{j+2}=(2),(9),(11),(12) \text { or (13) } \\
& Q_{j}=w_{1}^{-} w_{1} \mathbf{w}_{1}^{2} W_{j} \\
& Q_{j+1}=w_{0}^{4} w_{0}^{5} W_{j+1} \\
& Q_{j+2}=w_{1}^{-} w_{1} \mathbf{w}_{1}^{2} \\
& Q_{j}=w_{1}^{-} w_{1} w_{1} \mathbf{w}_{\mathbf{0}}^{\mathbf{3}} W_{j} \quad Q_{j+1}=w_{0}^{4} w_{0}^{2} w_{1}^{2} W_{j+1} \\
& Q_{j+2}=X_{j+2} w_{1} \mathbf{w}_{1}^{\mathbf{3}} W_{j+2}, X_{j+2} \in\left\{w_{1}^{-}, w_{1}\right\} \\
& Q_{j}=w_{1}^{-} w_{1} w_{1} w_{0}^{2} W_{j} \\
& Q_{j+1}=w_{0}^{4} w_{0}^{2} w_{1} W_{j+1} \\
& l_{Q_{j}} \equiv 0 \bmod v \\
& Q_{j+2}=X_{j+2} w_{1} w_{1} \mathbf{w}_{\mathbf{0}}^{\mathbf{5}} W_{j+2}, X_{j+2} \in\left\{w_{1}^{-}, w_{1}\right\} \\
& Q_{j}=w_{1}^{-} w_{1} w_{0}^{3} \\
& Q_{j+1}=w_{0}^{4} w_{1} \\
& Q_{j+2}=(1) \text { or }(7) \\
& Q_{j}=w_{1}^{-} w_{1} w_{0}^{2} \\
& Q_{j}=w_{1}^{-} w_{1} w_{0}^{5} \quad Q_{j+1}=w_{0}^{4} w_{1}^{2} \\
& Q_{j}=w_{1}^{-} w_{1} \mathbf{w}_{\mathbf{0}}^{\mathbf{6}} W_{j} \\
& Q_{j+1}=w_{0}^{4} w_{1}^{3} W_{j+1} \\
& Q_{j+2}=w_{1}^{3} w_{0}^{2} \\
& Q_{j+2}=(9) \\
& Q_{j+2}=w_{1}^{2} w_{0}^{5}=(2) \\
& Q_{j}=w_{1}^{-} w_{1} w_{0}^{5} \mathbf{w}_{\mathbf{1}} W_{j} \quad Q_{j+1}=w_{0}^{4} w_{1}^{2} w_{0}^{3} W_{j+1} \\
& Q_{j+2}=X_{j+2} w_{1} \mathbf{w}_{\mathbf{0}}^{\mathbf{8}} W_{j+2}, X_{j+2} \in\left\{w_{1}^{-}, w_{1}\right\} \\
& Q_{j+2}=X_{j+2} w_{1} w_{0}^{5} \mathbf{w}_{1}^{\mathbf{2}} W_{j+2}, X_{j+2} \in\left\{w_{1}^{-}, w_{1}\right\}
\end{aligned}
$$

Note that in each equation above $W_{j+1}$ and $W_{j+2}$ respectively contain at least the same number of words from the set $\left\{w_{0}, w_{1}^{-}, w_{1}, w_{0}^{2}, w_{0}^{3}\right\}$ as $W_{j}$ and $W_{j+1}$.

The same result can be proven for words $Q_{j+1}=X_{j+1} W_{j+1,1} w_{0}^{5} W_{j+1,2}$ by using the same method. The proof is left to the reader.

Case II.A.4. $l_{w_{1}}>5\left(w_{0}=1, \# 1=1\right)$.

Note that with $l_{w_{1}}>5, \# 1=1, w_{1}=0^{6} 0^{\# 0-6}$. Table 10 proves the case for $l_{w_{1}}=6$ :

Table 10: $w_{1}=0^{6}$

\begin{tabular}{|l|l|l||l||l||l||l||l||l||l|}
\hline & $w_{0}$ & $w_{1}$ & $w_{0}^{3}$ & $w_{1}^{2}$ & $w_{0}^{6}$ & $w_{1}^{3}$ & $\ldots$ & $w_{1}^{n}$ & $w_{0}^{3 n}$ \\
\hline$S_{0}$ & $w_{1}$ & $w_{0}^{3}$ & $w_{1}^{2}$ & $w_{0}^{6}$ & $w_{1}^{3}$ & $w_{0}^{9}$ & $\ldots$ & $w_{0}^{3 n}$ & $w_{1}^{\mid 3 n / 2\rceil}$ \\
$S_{1}$ & $\epsilon \checkmark$ & $w_{0}^{3}$ & $w_{1} \checkmark$ & $w_{0}^{6}$ & $w_{1}^{3}$ & $w_{0}^{9}$ & $\ldots$ & $w_{0}^{3 n}$ & $w_{1}^{\lfloor 3 n / 2\rfloor}$ \\
\hline
\end{tabular}


It immediately follows from Table 10 that $T$ will always have unbounded growth on any initial word $A_{0} \neq 0$ that results in the production of a word that contains $w_{1}^{2}$ (or $w_{1}^{-} w_{1}$ ) at least once. It can be easily checked that this is the case for any word $A_{0} \neq 0$ (Note that for every word $Q_{1}$ produced after one round on $A_{0} \neq 0, Q_{1}=1 W, Q_{1}=w_{1} W$ or $\left.Q_{1}=w_{1}^{-} W, W \in\left\{w_{0}, w_{1}\right\}^{*}\right)$.

It immediately follows from the generalization of Table 10 that any tag system $T$ with $l_{w_{1}}>6$ will always have unbounded growth on any initial word $A_{0} \neq 0$.

Case II.B. $\# 1=2\left(w_{0}=1\right)$.

We split the case into 6 main cases according to the value of $l_{w_{1}}$, i.e., $l_{w_{1}}=3, l_{w_{1}}=4, l_{w_{1}}=5, l_{w_{1}}=$ $6, l_{w_{1}}=7, l_{w_{1}}>7$. We do not consider cases with $l_{w_{1}} \leq 3$ since it follows from Wang's decidability criterion that for any tag systems $T$, if $l_{\max } \leq v$ then $T$ has a decidable reachability problem.

It follows from Corollary 1 that for each of the tag systems covered by this case, any word $Q_{j}, j \geq 2$ produced after $j$ rounds on $A_{0}$ :

$$
\text { (A) } Q_{j}=X_{j} W_{j}, X_{j} \in\left\{\epsilon, \vec{B}_{1}^{-}, w_{1}^{-}, \vec{A}_{1}^{-}\right\}, W_{j} \in\left\{\vec{B}_{1}, w_{1}, \vec{A}_{1}\right\}^{*}
$$

Case II.B.1. $l_{w_{1}}=3\left(w_{0}=1, \# 1=2\right)$

It can be determined for any tag system from this class that it will either halt or become periodic. There are three different tag systems covered by this case, i.e., either $w_{1}=100, w_{1}=010$ or $w_{1}=001$. In the following tables it is shown that the table method halts for all three tag systems, and it thus follows that all three tag systems will always halt or become periodic on any initial word.

Table 11: Case $w_{0}=1, w_{1}=100$

\begin{tabular}{|c|c|c|c|c|}
\hline & $w_{0}$ & $w_{1}$ & $w_{1} w_{0}$ & $w_{0} w_{1}$ \\
\hline$S_{0}$ & $w_{1}$ & $w_{1} w_{0}$ & $w_{1} w_{0} \checkmark$ & $w_{1} w_{0} \checkmark$ \\
$S_{1}$ & $\epsilon \checkmark$ & $w_{0} \checkmark$ & $w_{0} w_{1}$ & $w_{1} w_{0} \checkmark$ \\
\hline
\end{tabular}

Table 12: Case $w_{0}=1, w_{1}=010$

\begin{tabular}{|c|c|c|c|}
\hline & $w_{0}$ & $w_{1}$ & $w_{0} w_{0}$ \\
\hline$S_{0}$ & $w_{1}$ & $w_{0} w_{0}$ & $w_{1} \checkmark$ \\
$S_{1}$ & $\epsilon \checkmark$ & $w_{1} \checkmark$ & $w_{1} \checkmark$ \\
\hline
\end{tabular}

Table 13: Case $w_{0}=1, w_{1}=001$

\begin{tabular}{|c|c|c|c|c|}
\hline & $w_{0}$ & $w_{1}$ & $w_{0} w_{1}$ & $w_{1} w_{0}$ \\
\hline$S_{0}$ & $w_{1}$ & $w_{0} w_{1}$ & $w_{1} w_{0}$ & $w_{0} w_{1} \checkmark$ \\
$S_{1}$ & $\epsilon \checkmark$ & $w_{0} \checkmark$ & $w_{0} w_{1} \checkmark$ & $w_{0} w_{1} \checkmark$ \\
\hline
\end{tabular}


Case II.B.2. $l_{w_{1}}=4\left(w_{0}=1, \# 1=2\right)$.

There are exactly 4 tag systems $T_{1}-T_{4}$ covered by this case, i.e., $T_{1}$ with $w_{1,1}=1000, T_{2}$ with $w_{1,2}=$ 0100, $T_{3}$ with $w_{1,3}=0010$ and $T_{4}$ with $w_{1,4}=0001$. For each of these $T_{i}, T_{i}$ either produces $\vec{B}_{1}=11$ or $\vec{A}_{1, i}=1^{\left\lfloor r_{1} / 2\right\rfloor} w_{1} 1^{\left\lfloor t_{1} / 2\right\rfloor}$ after one $s$-round on $w_{1, i}$.

Let $A_{0}$ be some initial word. It can be proven that if $T_{i}$ does not result in the production of a word $Q_{j}$ that contains at least one word $\vec{A}_{1, i}$ or $\vec{A}_{1, i}^{-}$after at most 4 rounds on $A_{0}$ then $T$ is periodic on $A_{0}$. After two rounds on $A_{0}, T$ produces (Corollary 1 ):

$$
Q_{2}=X_{2} V_{2} \quad X_{2} \in\left\{\epsilon, \vec{B}_{1}^{-}, w_{1, i}^{-}, \vec{A}_{1, i}^{-}\right\}, V_{2} \in\left\{\vec{B}_{1}, w_{1, i}, \vec{A}_{1, i}\right\}^{*}
$$

If $Q_{2}$ does not contain any word $\vec{A}_{1, i}$ or $\vec{A}_{1, i}^{-}$then the parity of $l_{Q_{2}}$ is determined by $X_{2}$ since $l_{w_{1, i}}$ and $l_{\vec{B}_{1}}$ are even. I.e., $l_{Q_{2}}$ is even if $X_{2}=\epsilon$, or else, $l_{Q_{2}}$ is odd. After one more round on $Q_{2}, T$ produces:

$$
Q_{3}=X_{3} V_{3} \quad X_{3} \in\left\{\epsilon, \vec{B}_{1}^{-}, w_{1, i}^{-}, \vec{A}_{1, i}^{-}\right\}, V_{2} \in\left\{\vec{B}_{1}, w_{1, i}, \vec{A}_{1, i}\right\}^{*}
$$

If $Q_{3}$ does not contain the words $\vec{A}_{1, i}$ or $\vec{A}_{1, i}^{-}$then the parity of $l_{Q_{3}}$ is determined by $X_{2}$ in $Q_{2}$ since $l_{V_{2}}$ and $l_{V_{3}}$ are even and $X_{3}=\epsilon$ when $l_{Q_{2}}$ is even and $X_{3}$ is odd when $l_{Q_{2}}$ is odd. It now follows that if $Q_{2}$ and $Q_{3}$ do not contain any word $\vec{A}_{1, i}$ or $\vec{A}_{1, i}^{-}$then $Q_{4}=Q_{2}$. In order to see this note that every word $w_{1, i}$ and $\vec{B}_{1}$ in $Q_{2}$ is entered with the same shift $s\left(0\right.$, when $X_{2}=\epsilon, 1$ otherwise) resulting in $\vec{B}_{1}$ and $w_{1, i}$ in $Q_{3}$ respectively. We also have that each word $\vec{B}_{1}$ and $w_{1, i}$ in $Q_{3}$ will be entered with the same shift $s$ resulting in $w_{1, i}$ and $\vec{B}_{1}$ respectively in $Q_{4}$ and $X_{4}=X_{2}$. We thus have that $Q_{2}=Q_{4}$.

We will now prove that $T_{i}$ always has unbounded growth on any word $Q_{j}$ that contains $\vec{A}_{1, i}$ or $\vec{A}_{1, i}^{-}$at least once by applying Lemma 2 . Note first of all that condition c is met. I.e., for any such word $Q_{j}$ every subword in $Q_{j}$ from the set $\left\{\vec{B}_{1}, w_{1, i}, \vec{A}_{1, i}, \vec{B}_{1}^{-}, w_{1, i}^{-}, \vec{A}_{1, i}^{-}\right\}$will result in the production of at least one word from that same set in $Q_{j+1}$.

Now, for each $T_{i}$ we have the following words $\vec{A}_{1, i}: \vec{A}_{1,1}=\vec{A}_{1,2}=w_{1} 1, \vec{A}_{1,3}=\vec{A}_{1,4}=1 w_{1}$. After one $s$-round on $\vec{A}_{1,1}$ and $\vec{A}_{1,4} T$ produces either $\vec{A}_{2,1}=\vec{A}_{1,1} w_{1,1}$ and $\vec{A}_{2,4}=w_{1,4} \vec{A}_{1,4}$, respectively, or $\vec{B}_{2,1}=\vec{B}_{2,4}=\vec{B}_{1}$. After one $s$-round on $\vec{A}_{1,2}$ and $\vec{A}_{1,3} T$ produces either $\vec{A}_{2,2}=\vec{B}_{1} w_{1,2}$ and $\vec{A}_{2,3}=w_{1,3} \vec{B}_{1}$ or $\vec{B}_{2,2}=\vec{A}_{1,2}$ and $\vec{B}_{2,3}=\vec{A}_{1,3}$. Since each $l_{\vec{A}_{1, i}}$ is odd, it now easily follows that for any word $Q_{j}$ that contains at least two words $\vec{A}_{1, i}$ (or one word $\vec{A}_{1, i}^{-}$and at least one word $\vec{A}_{1, i}$ ) there is always at least one word $\vec{A}_{1, i}$ in $Q_{j}$ that results in two words from the set $\left\{\vec{B}_{1}, w_{1, i}, \vec{A}_{1, i}, \vec{B}_{1}^{-}, w_{1, i}^{-}, \vec{A}_{1, i}^{-}\right\}$in $Q_{j+1}$ and $Q_{j+1}$ will again contain at least one word $\vec{A}_{1, i}$. The reason for this is that the only words between two consecutive words $\vec{A}_{1, i}$ are words $\vec{B}_{1}$ and words $w_{1, i}$. This means that every two words $\vec{A}_{1, i}$ in $Q_{j}$ are separated by a subword of even length and thus, when the first $\vec{A}_{1, i}$ is entered with shift 0 , the second will be entered with shift 1 and vice versa. It thus follows that at least one word $\vec{A}_{1, i}$ in $Q_{j}$ will result in $\vec{A}_{2, i}$ in $Q_{j+1}$ (Note that $\vec{A}_{2, i}$ is different from any words in the set $\left\{\vec{B}_{1}, w_{1, i}, \vec{A}_{1, i}, \vec{B}_{1}^{-}, w_{1, i}^{-}, \vec{A}_{1, i}^{-}\right\}$). Thus conditions a, b and c of Lemma 2 are met for words $Q_{j}$ that contain $\vec{A}_{1, i}$ at least twice (or one word $\vec{A}_{1, i}^{-}$ and at least one word $\vec{A}_{1, i}$ ). Furthermore, $Q_{j+1}$ contains at least one word $\vec{A}_{1, i}$ (or $\vec{A}_{1, i}^{-}$).

The same can be proven if $Q_{j}$ contains only one word $\vec{A}_{1, i}$ or $\vec{A}_{1, i}$. We can furthermore prove that for 
these words $Q_{j}$ property $\mathrm{d}$ is met. We will only prove the result for $T_{1}$. The proofs for $T_{2}-T_{4}$ follow the same method.

Note that $T_{1}$ produces $\overrightarrow{A_{1,1}}$ after one 0 -round and $\vec{B}_{1}$ after one 1 -round on $w_{1,1}$. Now, given a word $Q_{j}$ that contains only one word $\overrightarrow{A_{1,1}}$ (or one word $\vec{A}_{1,1}^{-}$), then:

$$
Q_{j}=X_{j} V_{j} \vec{A}_{1,1} W_{j}\left(\text { or } Q_{j}=\vec{A}_{1,1}^{-} W_{j}\right) \quad X_{j} \in\left\{\epsilon, \vec{B}_{1}^{-}, w_{1,1}^{-}\right\}, V_{j}, W_{j} \in\left\{\vec{B}_{1}, w_{1,1}\right\}^{*}
$$

If $X_{j}=\epsilon$ and $Q_{j} \neq \vec{A}_{1,1}^{-} W_{j}$ then $T$ produces the word $\vec{A}_{2,1}=\vec{A}_{1,1} w_{1,1}$ in $Q_{j+1}$ and thus conditions a, b,c and d of Lemma 2 are met. I.e., there is at least one subword in $Q_{j}$ from the set $\left\{\vec{A}_{1,1}, \vec{B}_{1}, w_{1,1}, \vec{A}_{1,1}^{-}, \vec{B}_{1}^{-}, w_{1,1}^{-}\right\}$ from which $T$ produces a new word that is the concatenation of two words from that same set in $Q_{j+1}$, this word is different from every word from that set and $Q_{j+1}$ again contains at least one word $\vec{A}_{1,1}$. If $\vec{A}_{1,1}$ in $Q_{j}$ is entered with shift 1 then $l_{Q_{j}}$ is even. This means that after one round on $Q_{j} T$ produces:

$$
Q_{j+1}=V_{j+1} \vec{B}_{1} W_{j+1} \quad V_{j+1} \in\left\{\vec{B}_{1}, w_{1,1}\right\}^{*}, W_{j+1} \in\left\{w_{1,1}, \vec{A}_{1,1}\right\}
$$

If $W_{j}$ contains at least one word $w_{1,1}$ then $W_{j+1}$ must contain at least one word $\vec{A}_{1,1}$ (Note that if $\vec{A}_{1,1}$ in $Q_{j}$ is entered with shift 1 then the leftmost $w_{1,1}$ in $W_{j}$ is entered with shift 0 ). It then easily follows that $T_{1}$ will produce at least one word $\vec{A}_{2,1}$ in $Q_{j+2}$ since the leftmost word $\vec{A}_{1,1}$ in $Q_{j+1}$ will be entered with shift 0 and thus conditions a-d of Lemma 2 are met.

If $W_{j+1}$ contains no word $\vec{A}_{1,1}$ then $l_{Q_{j+1}}$ is even. In that case, if $Q_{j+1}$ contains at least one word $w_{1,1}$ then it easily follows that it takes two more rounds on $Q_{j+1}$ to produce at least one word $\overrightarrow{A_{2,1}}$ in $Q_{j+3}$ (Note that since $l_{Q_{j+1}}$ is even, the first word $\vec{A}_{1,1}$ produced in $Q_{j+2}$ will be entered with shift 0 ). Finally, if $Q_{j+1}$ contains no word $w_{1,1}$ then, $Q_{j+1}=\vec{B}_{1}^{n}, n \geq 1$ and it easily follows that it takes three more rounds on $Q_{j+1}$ to produce at least one word $\vec{A}_{2,1}$ in $Q_{j+4}$. Clearly, also in these last cases conditions a-d of Lemma 2 are met.

The result of unbounded growth now easily follows for words $Q_{j}$ that contain $\vec{A}_{1,1}$ or $\vec{A}_{1,1}^{-}$at least once since any such word always satisfies conditions a-d of Lemma 2.

Case II.B.3. $l_{w_{1}}=5\left(w_{0}=1, \# 1=2\right)$.

There are exactly 5 tag systems $T_{5}-T_{9}$ covered by this case. For any $T_{i}, w_{1, i}=0^{r_{1}} 10^{t_{1}}$ and after one $s$-round on $w_{1, i} T_{i}$ either produces $\vec{B}_{1}=1^{\left\lceil r_{1}\right\rceil+\left\lceil t_{1}\right\rceil}$ or $\vec{A}_{1, i}=1^{\left\lfloor r_{1} / 2\right\rfloor} w_{1} 1^{\left\lfloor t_{1} / 2\right\rfloor}$. We split the case according to the value of $\left\lceil r_{1} / 2\right\rceil+\left\lceil t_{1} / 2\right\rceil$, i.e., either $\left\lceil r_{1} / 2\right\rceil+\left\lceil t_{1} / 2\right\rceil=2$ or $\left\lceil r_{1} / 2\right\rceil+\left\lceil t_{1} / 2\right\rceil=3$.

Note that for each of these $T_{i}$, any word $Q_{j}, j \geq 2$ is of the form (A).

Case II.B.3.a. $\vec{B}_{1}=11\left(w_{0}=1, \# 1=2, l_{w_{1}}=5\right)$.

There are exactly 3 tag systems covered by this case, i.e., $T_{5}$ with $w_{1,5}=10000, T_{6}$ with $w_{1,6}=00100$ and $T_{7}$ with $w_{1,7}=00001$. It is easily proven for each of these tag systems that for any word $A_{0} \neq 0$ it takes at most 6 rounds on $A_{0}$ to produce a word $Q_{j}$ that contains at least one word $\vec{A}_{1, i}$ or $\vec{A}_{1, i}^{-}$. The reason for this is that $l_{w_{1}}$ is odd (note that if $Q_{2}$ contains at least two words $\vec{B}_{1}$ or $w_{1, i}$ then it easily follows that it takes at most two rounds on $Q_{2}$ to produce a word that contains at least one word $\vec{A}_{1, i}$ or $\left.\vec{A}_{1, i}^{-}\right)$.

We prove the case by showing that all conditions of Lemma 2 are met for any word $Q_{j}, j \geq 2$ of form (A) that contains at least one word $\vec{A}_{1, i}$ or $\vec{A}_{1, i}^{-}$. First of all, note that it easily follows that condition (c) is 
satisified, i.e., every word from the set $\left\{\vec{B}_{1}^{-}, w_{1}^{-}, \vec{A}_{1}^{-}, \vec{B}_{1}, w_{1}, \vec{A}_{1}\right\}$ will again result in at least one word from that same set in $Q_{j+1}$.

The values for the words $\vec{A}_{1, i}$ are $\vec{A}_{1,5}=w_{1,5} \vec{B}_{1}, \vec{A}_{1,6}=1 w_{1,6} 1, \vec{A}_{1,7}=\vec{B}_{1} w_{1,7}$. Now, for any word $Q_{j}=\vec{A}_{1, i}$ or $Q_{j}=\vec{A}_{1, i}^{-}$it can be easily computed that it takes at most 2 rounds of $T$ on $Q_{j}$ to produce a word that again contains $\vec{A}_{1, i}$ and at least one more word from the set $\left\{\vec{B}_{1}^{-}, w_{1}^{-}, \vec{A}_{1}^{-}, \vec{B}_{1}, w_{1}, \vec{A}_{1}\right\}$. This means that we only need to consider words $Q_{j}$ that contain either one of the following words as a subword:

$$
\begin{aligned}
& (a)=\vec{B}_{1} \vec{A}_{1, i}\left(\text { or } \vec{B}_{1}^{-} \vec{A}_{1, i}\right) \\
& (b)=\vec{A}_{1, i} \vec{B}_{1}\left(\text { or } \vec{A}_{1, i}^{-} \vec{B}_{1}\right. \\
& (c)=w_{1, i} \vec{A}_{1, i}\left(\text { or } w_{1, i}^{-} \vec{A}_{1, i}\right) \\
& (d)=\vec{A}_{1, i} w_{1, i}\left(\text { or } \vec{A}_{1, i}^{-} w_{1, i}\right. \\
& (e)=\vec{A}_{1, i}^{2}\left(\text { or } \vec{A}_{1, i}^{-} \vec{A}_{1, i}\right)
\end{aligned}
$$

For any tag system $T_{5}-T_{7}$ it can be easily checked that if $Q_{j}$ contains at least one of the subwords $(a)-(e)$ then it takes at most three rounds of $T$ on $Q_{j}$ to produce from each of these subwords again one of the words $(a)-(e)$ plus at least one word from the set $\left\{\vec{B}_{1}, w_{1, i}, \vec{A}_{1, i}, \vec{B}_{1}^{-}, w_{1, i}^{-}, \vec{A}_{1, i}^{-}\right\}$in the word $Q_{j+i}, 0<i \leq 3$. It thus easily follows that conditions a-d of Lemma 2 are satisfied and the result of unbounded growth immediately follows.

Case II.B.3.b. $\vec{B}_{1}=111\left(w_{0}=1, \# 1=2, l_{w_{1}}=5\right)$.

There are 2 tag systems covered by this case, i.e., $T_{8}$ with $w_{1,8}=01000, T_{9}$ with $w_{1,9}=00010$. Now, given some initial word $A_{0} \neq 0$ it is easily checked that either $T_{i}$ is periodic on $A_{0}$ or it takes at most 4 rounds on $A_{0}$ to produce a word that contains at least one word $\overrightarrow{A_{1, i}}$ or $\vec{A}_{1, i}^{-}$. Both tag systems are periodic on $w_{1, i}$ and 11 .

We will now prove that $T_{i}$ always has unbounded growth on any word $Q_{j}$ that contains $\vec{A}_{1, i}$ or $\vec{A}_{1, i}^{-}$ at least once by applying Lemma 2. The main difficulty with these two tag systems is that after one $s$-round on $\vec{A}_{1, i}\left(\vec{A}_{1,8}=w_{1,8} 1, \vec{A}_{1,9}=1 w_{1,9}\right) T_{i}$ either produces a word $\vec{A}_{2, i}$ that contains $w_{1}$ and $\vec{A}_{1, i}$ or $\vec{B}_{1}=111$. We will only consider $T_{8}$ since $w_{1,9}$ is the mirror image of $w_{1,8}$ and the proof of $T_{9}$ thus easily reduces to the proof of $T_{8}$.

Now, it is easily computed that for any word $Q_{j}=\vec{A}_{1,8}$ or $Q_{j}=\vec{A}_{1,8}^{-}$it takes at most 3 rounds of $T_{8}$ on $Q_{j}$ to produce a word that contains $\vec{A}_{1,8}^{-}$and either $\vec{B}_{1}$ or $w_{1,8}$. We thus only need to consider words $Q_{j}$ that contains one of the words $(a)-(e)$ with $i=8$ as a subword.

It can be easily verified that if $Q_{j}$ contains at least one of the subwords $(b)-(e)$ then it takes at most three rounds on $Q_{j}$ to produce from any of these subwords $(b)-(e)$ again one of he words $(a)-(e)$ and at least one more word from the set $\left\{\vec{B}_{1}, w_{1,8}, \vec{A}_{1,8}, \vec{B}_{1}^{-}, w_{1,8}^{-}, \vec{A}_{1,8}^{-}\right\}$in the word $Q_{j+i}, 0<i \leq 3$. The problematic cases are words that contain only one word $\vec{A}_{1, i}$ and $\vec{A}_{1, i}$ is preceded by $\vec{B}_{1}$. There is no problem when $(a)$ is entered with shift 0 : after one 0-round on $(a) T_{8}$ produces $w_{1,8}^{2} \vec{A}_{1,8} w_{1,8}$. However, when $(a)$ is entered with shift 1 , then $T_{8}$ produces $w_{1,8} \vec{B}_{1}$, after one 0 -round on $w_{1,8} \vec{B}_{1} T_{8}$ produces $\vec{B}_{1} w_{1,8}$ and after one 1-round on $\vec{B}_{1} w_{1,8} T_{8}$ again produces $w_{1,8} \vec{B}_{1}$. This means that there is not necessarily an $n$ such that the subword $(a)$ results again in one of the words $(a)-(e)$ and at least one more subword from the set $\left\{\vec{B}_{1}, w_{1,8}, \vec{A}_{1,8}, \vec{B}_{1}^{-}, w_{1,8}^{-}, \vec{A}_{1,8}^{-}\right\}$in $Q_{j+n}$ so we need to consider this special case separately. 
Now, if $Q_{j}=(a)^{-}$then it can be easily checked that $Q_{j+3}=w_{1,8}^{2} \vec{A}_{1,8}$. If $Q_{j} \neq(a)^{-}$but contains (a) and $(a)$ is followed by any word $\vec{B}_{1}, w_{1,8}$ or $\vec{A}_{1,8}$ or $(a)$ is preceded by $\vec{A}_{1,8}$ then $Q_{j}$ contains at least one of the words $(b)-(e)$. If $(a)$ is preceded by $\vec{B}_{1}$ then it is easily computed that it takes at most two rounds on $Q_{j}$ to produce from $\vec{B}_{1}^{2} \vec{A}_{1,8}$ one word $(a)-(e)$ and at least one word from the set $\left\{\vec{B}_{1}, w_{1,8}, \vec{A}_{1,8}, \vec{B}_{1}^{-}, w_{1,8}^{-}, \vec{A}_{1,8}^{-}\right\}$. A similar result is easily proven when $Q_{j}$ contains $(a)$ and $\vec{B}_{1}^{2}$ (note that after one $s$-round of $T_{8}$ on $\vec{B}_{1}^{2} T_{8}$ produces $w_{1,8}^{3}$ ). The only remaining possibilities are that $Q_{j}$ is one of the following words:

$$
\begin{array}{ll}
Q_{j}=X_{j} w_{1}^{n_{1}}\left(\vec{B}_{1} w_{1,8}\right)^{n_{1}} \ldots w_{1}^{n_{i}}\left(\vec{B}_{1} w_{1,8}\right)^{n_{i}} \vec{B}_{1} \vec{A}_{1,8} & X_{j} \in\left\{\epsilon, \vec{B}_{1}^{-} w_{1,8}, w_{1,8}^{-}\right\}, n_{j} \in \mathbb{N} \\
Q_{j}=X_{j} w_{1}^{n_{1}}\left(w_{1,8} \vec{B}_{1}\right)^{n_{1}} \ldots w_{1}^{n_{i}}\left(w_{1,8} \vec{B}_{1}\right)^{n_{i}} \vec{A}_{1,8} & X_{j} \in\left\{\epsilon, w_{1,8}^{-} \vec{B}_{1}, w_{1,8}^{-}\right\}, n_{j} \in \mathbb{N}
\end{array}
$$

It can be easily checked for each of these possibilities that it takes at most 3 rounds to produce a word $Q_{j+i}, 0<i \leq 3$ such that $Q_{j+i}$ contains at least one of the words $(b)-(e)$. It now easily follows from Lemma 2 that $T_{8}$ always has unbounded growth on any word $A_{0} \neq 0$ for which $T_{8}$ produces a word that contains at least one word $\vec{A}_{1, i}$ or $\vec{A}_{1, i}^{-}$.

Case II.B.4. $l_{w_{1}}=6\left(w_{0}=1, \# 1=2\right)$.

There are 6 tag systems $T_{10}-T_{15}$ covered by this case, i.e., $T_{10}$ with $w_{1,10}=100000$ and $T_{11}$ with $w_{1,11}=010000, T_{12}$ with $w_{1,12}=001000, T_{13}$ with $w_{1,13}=000100, T_{14}$ with $w_{1,14}=000010, T_{15}$ with $w_{1,15}=000001$. For each of these $T_{i}, T_{i}$ either produces $\vec{B}_{1}=111$ or $\vec{A}_{1, i}=1^{\left\lfloor r_{1} / 2\right\rfloor} w_{1} 1^{\left\lfloor t_{1} / 2\right\rfloor}$ after one $s$-round on $w_{1, i}$. For each of these $T_{i}, T_{i}$ either produces $\vec{B}_{1}=111$ or $\vec{A}_{1, i}=1^{\left\lfloor r_{1} / 2\right\rfloor} w_{1} 1^{\left\lfloor t_{1} / 2\right\rfloor}$ after one $s$-round on $w_{1, i}$. Also, every word $Q_{j}$ is a word of the form (A). From this, it easily follows that condition c of Lemma 2 is satisfied. I.e., for any of the subwords in $Q_{j}$ from the set $\left\{\vec{B}_{1}^{-}, w_{1}^{-}, \vec{A}_{1}^{-}, \vec{B}_{1}, w_{1}, \vec{A}_{1}\right\}$ $T_{i}$ again produces a word from that same set in $Q_{j+1}$. We can also prove that all the other conditions of Lemma 2are met.

It is easily proven that $T_{10}-T_{15}$ always have unbounded growth on any word $Q_{j}$ that contains one of the subwords $(a)-(e), 10 \leq i \leq 15$ or:

$$
\begin{aligned}
& (f) w_{1, i}^{2}\left(\text { or } w_{1, i}^{-} w_{1, i}\right) \\
& (g) w_{1, i} \vec{B}_{1}\left(\text { or } w_{1, i}^{-} \vec{B}_{1}\right) \\
& (h) \vec{B}_{1} w_{1, i}\left(\text { or } \vec{B}_{1}^{-} w_{1, i}\right) \\
& (i) \\
& \vec{B}_{1}^{2}\left(\text { or } \vec{B}_{1}^{-} \vec{B}_{1}\right)
\end{aligned}
$$

Indeed, it can be verified that if one of these subwords $(a)-(i)$ is in $Q_{j}$ then it takes at most two rounds on $Q_{j}$ to produce from one of these subwords $(a)-(i)$ again one of these subwords and at least one more word from the set $\left\{\vec{B}_{1}, w_{1, i}, \vec{A}_{1, i}, \vec{B}_{1}^{-}, w_{1, i}^{-}, \vec{A}_{1, i}^{-}\right\}$in the word $Q_{j+i}, 0<i \leq 2$.

It is furthermore easily proven that for any word $A_{0} \neq 0$ it takes but a finite number of rounds of $T_{i}$ on $A_{0}$ to produce a word that contains at least one of the subwords $(a)-(i)$. Hence it easily follows that each $T_{i}$ always has unbounded growth on any initial word $A_{0}$.

Case II.B.5. $l_{w_{1}}=7\left(w_{0}=1, \# 1=2\right)$.

There are 7 tag systems covered by this case. For any $T_{i}, w_{1, i}=0^{r_{1}} 10^{t_{1}}$ and after one $s$-round on $w_{1, i}$ $T_{i}$ either produces $\vec{B}_{1}=1^{\left\lceil r_{1}\right\rceil+\left\lceil t_{1}\right\rceil}$ or $\vec{A}_{1, i}=1^{\left\lfloor r_{1} / 2\right\rfloor} w_{1} 1^{\left\lfloor t_{1} / 2\right\rfloor}$. We split the case according to the value 
of $\left\lceil r_{1} / 2\right\rceil+\left\lceil t_{1} / 2\right\rceil$, i.e., either $\left\lceil r_{1} / 2\right\rceil+\left\lceil t_{1} / 2\right\rceil=3$ or $\left\lceil r_{1} / 2\right\rceil+\left\lceil t_{1} / 2\right\rceil=4$

Case II.B.5.a. $\vec{B}_{1}=111\left(w_{0}=1, \# 1=2, l_{w_{1}}=7\right)$.

There are 4 tag systems covered by this case, i.e., $T_{16}, w_{1,16}=1000000, T_{17}$ with $w_{1,17}=0010000$, $T_{18}$ with $w_{1,18}=0000100$ and $T_{19}$ with $w_{1,19}=0000001$. These tag systems always have unbounded growth on any initial word $A_{0} \neq 0$. The proof is similar to the proof of unbounded growth for $T_{10}-T_{15}$ and thus we leave it to the reader.

Case II.B.5.b. $\vec{B}_{1}=1111\left(w_{0}=1, \# 1=2, l_{w_{1}}=7\right)$.

There are exactly 3 tag systems $T_{20}-T_{22}$ for which $\vec{B}_{1}=1111$, i.e., $T_{20}$ with $w_{1,20}=0100000, T_{21}$ with $w_{1,21}=0001000$ and $T_{22}=0000010$. After one $s$-round on $w_{1, i}$ each of these $T_{i}$ either produces $\vec{B}_{1}=1111$ or $\vec{A}_{1, i}=1^{\left\lfloor r_{1} / 2\right\rfloor} w_{1} 1^{\left\lfloor t_{1} / 2\right\rfloor}$. It is easily proven that every $T_{i}$ has unbounded growth on any word $A_{0} \neq 0$. The reason for this is that, on the one hand, it easily follows that $l_{\vec{A}_{1, i}}>l_{w_{1, i}}$ and, on the other hand, every word $\vec{B}_{1}$ produced from one word $w_{1, i}$ always results in the production of two words $w_{1, i}$, whatever shift $\vec{B}_{1}$ is entered with. Thus, once $w_{1}$ is produced from the initial word $A_{0}$ then $T$ must have unbounded growth on $A_{0}$. It can be easily checked that it takes at most 2 rounds on any initial word $A_{0} \neq 0$ to produce a word that contains $w_{1}$ at least once, hence the result of unbounded growth.

Case II.B.6. $w_{1}>7\left(w_{0}=1, \# 1=2\right)$.

Let $T$ be a tag system covered by Case II.B.6. After one $s$-round on $w_{1} T$ either produces $\vec{B}_{1}=1^{n}$ or $\vec{A}_{1, i}=1^{\left\lfloor r_{1} / 2\right\rfloor} w_{1} 1^{\left\lfloor t_{1} / 2\right\rfloor}$. However, since $l_{w_{1}}>7$ and $w_{1}$ contains only one 1 symbol, it easily follows that $n \geq 4$ in $\vec{B}_{1}=1^{n}$. The result of unbounded growth thus easily follows (See Case II.B.5.b).

Case II.C. $\# 1>2\left(w_{0}=1\right)$.

We split the case into two main cases according to the value of $l_{w_{1}}$, i.e., $l_{w_{1}}=3$ and $l_{w_{1}}>3$. We do not take into account the case with $l_{w_{1}}<3$ due to Wang's decidability criterion.

Case II.C.1. $l_{w_{1}}=3\left(\# 1>2, w_{0}=1\right)$.

There are exactly four tag systems in this class depending on the value of $w_{1}$, i.e., $w_{1}=111, w_{1}=$ $101, w_{1}=110$ and $w_{1}=011$. We need to study each of these tag systems separately.

Case II.C.1.a. $w_{1}=111\left(l_{w_{1}}=3, \# 1>2, w_{0}=1\right)$.

This tag system $T$ will always have unbounded growth on any word $A_{0} \neq 0$. The reason for this is that for this case any word $Q_{j}$ produced after $j$ rounds on $A_{0}$ consists entirely of 1 symbols and with every computation step, two 1 symbols are appended and only 1 deleted.

Case II.C.1.b. $w_{1}=101\left(l_{w_{1}}=3, \# 1>2, w_{0}=1\right)$.

We will prove that this tag system always has unbounded growth on any initial word $A_{0} \neq 0$. Note that $T$ either reads two 1 symbols or one 0 during one $s$-round on $w_{1}$. In order to prove the case we apply the table method for 2 iterations on $w_{1}$ :

Table 14: $w_{1}=101$ 


\begin{tabular}{|l|l||l||l|l||}
\hline & $w_{1}$ & $\overrightarrow{A_{1}}$ & $\overrightarrow{A_{2}}$ & $\vec{B}_{2}$ \\
\hline$S_{0}$ & $\overrightarrow{A_{1}}=w_{1}^{2}$ & $\overrightarrow{A_{2}}=\vec{A}_{1} \vec{B}_{1}$ & $\overrightarrow{A_{3}}=\vec{A}_{2} w_{1}$ & $\vec{B}_{3}=w_{1} \vec{B}_{2}$ \\
$S_{1}$ & $\vec{B}_{1}=w_{0} \checkmark$ & $\vec{B}_{2}=\vec{B}_{1} \vec{A}_{1}$ & $\vec{B}_{2} \checkmark$ & $\vec{A}_{2} \checkmark$ \\
\hline
\end{tabular}

It is easily checked that $T$ is periodic on the words $w_{1}^{-}$and $\vec{B}_{1}$. It can also be checked that it takes at most 3 rounds on any initial word $A_{0}$ that differs from these two periodic words and $A_{0} \neq 0$ before $T$ produces a word that contains $\vec{A}_{1}$ or $\vec{A}_{1}^{-}$at least once. Since it follows from Table 14 that after one $s$-round on $\vec{A}_{1} T$ produces either $\vec{A}_{2}$ or $\vec{B}_{2}$ and after one $s$-round of $T$ on $\vec{A}_{2}$ or $\vec{B}_{2} T$ always produces a word that contains either $\vec{A}_{2}$ or $\vec{B}_{2}$ it follows that any word $Q_{j}, j>3$ always contains $\vec{A}_{2}, \vec{A}_{2}^{-}, \vec{B}_{2}$ or $\vec{B}_{2}^{-}$at least once. Furthermore, for any such word $Q_{j}, j>3$ it is impossible that $Q_{j}$ contains $w_{0}^{2}$ as a subword. I.e., there is no combination of words $w_{1}$ and $w_{0}$ that results in $w_{0}^{2}$ (Note that the only way to produce $w_{0}$ is when $w_{1}$ is entered with shift 1$)$. We can now define $Q_{j}$ as follows:

$$
\begin{array}{cl}
Q_{j}=X_{j} V_{j} Y_{j} \quad X_{j} \in\left\{w_{1}^{-} w_{0}, w_{1}^{-} w_{1}, \epsilon\right\}, Y_{j} \in\left\{w_{0}, w_{1}, \epsilon\right\} \\
V_{j} \in\left\{w_{0} w_{1}, w_{1} w_{0}, w_{1}^{2}, \vec{A}_{2}, \vec{B}_{2}\right\}^{*}
\end{array}
$$

with the following restrictions on $X_{j}, V_{j}$ and $Y_{j}$ : (1) if $X_{j}=w_{1}^{-} w_{0}$ then the left end of $V_{j}$ cannot be $w_{0} w_{1}$ or $\vec{B}_{2}$ (2) no subword $\vec{B}_{2}$ or $w_{0} w_{1}$ in $V_{j}$ can be preceded by $\vec{A}_{2}$ or $w_{1} w_{0}$ and, finally (3) if $Y_{j}$ is $w_{0}$ then the right end of $V_{j}$ cannot be $\overrightarrow{A_{2}}$ or $w_{1} w_{0}$.

We will now prove that $T$ always has unbounded growth on any such word $Q_{j}$. In order to prove this, first of all note that after one round on $Q_{j}$ every one of the subwords in $X_{j} V_{j}$, i.e., words from the set $\left\{w_{1}^{-} w_{0}, w_{1}^{-} w_{1}, w_{0} w_{1}, w_{1} w_{0}, w_{1}^{2}, \vec{A}_{2}, \vec{B}_{2}\right\}$, results in at least one subword from that same set in $Q_{j+1}$ which is at least as long as the original word in $Q_{j}$. Furthermore, $Q_{j+1}$ is of the same form as $Q_{j}$ again containing at least one word $\vec{A}_{2}, \vec{A}_{2}^{-}, \vec{B}_{2}$ or $\vec{B}_{2}^{-}$. This follows from the productions of table 14 . It also follows immediately that the length of $Y_{j}$, which is either equal to $w_{0}, w_{1}$ or $\epsilon$, is bounded.

Now, if $Q_{j}$ is one of the words $\vec{A}_{2}, \vec{A}_{2}^{-}, \vec{B}_{2}$ or $\vec{B}_{2}^{-}$then it is easily checked that it takes at most 2 rounds of $T$ on $Q_{j}$ to produce a word that again contains $\vec{A}_{2}, \vec{A}_{2}^{-}$or $\vec{B}_{2}$ or $\vec{B}_{2}^{-}$and at least one word $w_{0}$ or $w_{1}$. This means that we only need to study words $Q_{j}$ that contain at least one of the following words as a subword:

$$
\begin{array}{llll}
\vec{A}_{2} w_{0}=w_{1}^{2} w_{0}^{2} & w_{0} \vec{A}_{2}=w_{0} w_{1}^{2} w_{0} & \vec{A}_{2}^{-} w_{0}=w_{1}^{-} w_{1} w_{0}^{2} & \\
\vec{A}_{2} w_{1}=w_{1}^{2} w_{0} w_{1} & w_{1} \vec{A}_{2}=w_{1}^{3} w_{0} & \vec{A}_{2}^{-} w_{1}=w_{1}^{-} w_{1} w_{0} w_{1} & w_{1}^{-} \vec{A}_{2}=w_{1}^{-} w_{1}^{2} w_{0} \\
\vec{B}_{2} w_{0}=w_{0} w_{1}^{2} w_{0} & w_{0} \vec{B}_{2}=w_{0}^{2} w_{1}^{2} & \vec{B}_{2}^{-} w_{0}=w_{1}^{2} w_{0} & \\
\vec{B}_{2} \vec{w}_{1}=w_{0} w_{1}^{3} & \vec{w}_{1} \vec{B}_{2}=w_{1} w_{0} w_{1}^{2} & \vec{B}_{2}^{-} w_{1}=w_{1}^{3} & w_{1}^{-} \vec{B}_{2}=w_{1}^{-} w_{0} w_{1}^{2}
\end{array}
$$

It is easily checked that if $Q_{j}$ contains any of the subwords (1)-(4) then it takes at most 3 rounds of $T$ on $Q_{j}$ to produce from any of these subwords again one of the words (1)-(4) and at least one more subword from the set $\left\{w_{1}, \vec{B}_{1} w_{1}, w_{1} \vec{B}_{1}\right\}$ (Note that the words $\vec{A}_{2} w_{0}, \vec{A}_{2}^{-} w_{0}$ and $w_{0} \vec{B}_{2}$ contain $w_{0}^{2}$ as a subword, so we do not need to take them into account. If $Q_{j}$ contains $\overrightarrow{A_{2}^{-}} w_{1}, w_{1}^{-} \overrightarrow{A_{2}}, \overrightarrow{B_{2}^{-}} w_{0}, \vec{B}_{2}^{-} w_{1}$ or $w_{1}^{-} \vec{B}_{2}$ these words are at the left end of $Q_{j}$ and are thus entered with shift 0 ). 
It now easily follows that $T$ always has unbounded growth on any word $Q_{j}$ that contains one of the words (1)-(4) at least once.

Case II.C.1.c. $w_{1}=110\left(l_{w_{1}}=3, \# 1>2, w_{0}=1\right)$.

It can be proven that this tag system is periodic on any word $A_{0} \neq 0$ from which $T$ produces $\left(w_{1} 1\right)^{n}$ after one round and has unbounded growth on any other initial word $A_{0} \neq 0$. Note that $T$ always reads one symbol 1 during one $s$-round on $w_{1}$. This immediately implies that $T$ cannot halt on any word $A_{0}$ that results in the production of a word that contains at least one $w_{1}$ or one $w_{1}^{-}$. It can be easily checked that it takes at most two rounds of $T$ on $A_{0} \neq 0$ to produce a word that contains $w_{1}$, thus $T$ can never halt on any word $A_{0} \neq 0$. In order to prove the case, we need to apply the table method to $w_{1}$.

Table 15: $w_{1}=110$

\begin{tabular}{|c|l||l||}
\hline & $w_{1}$ & $\vec{A}_{1}$ \\
\hline$S_{0}$ & $\overrightarrow{A_{1}}=w_{1} 1$ & $\vec{A}_{1} \checkmark$ \\
$S_{1}$ & $\vec{B}_{1}=w_{1} \checkmark$ & $\vec{B}_{2}=w_{1}^{2}$ \\
\hline
\end{tabular}

Given an initial word $A_{0} \neq 0$, then it follows from Table 15 and Corollary 1 that any word $Q_{j}, j>1$ :

$$
Q_{j}=X_{j} V_{j} \quad X_{j} \in\left\{\epsilon, w_{1}^{-}, \vec{B}_{2}^{-}, \vec{A}_{1}^{-}\right\}, V_{j} \in\left\{w_{1}, \vec{B}_{2}, \vec{A}_{1}\right\}^{*}
$$

If $Q_{j}=\vec{A}_{1}^{n}, n \in \mathbb{N}$ then $Q_{j}$ is periodic. The reason for this is that $\vec{A}_{1}$ reproduces itself when entered with shift 0 and $l_{\vec{A}_{1}}$ is even.

We can now prove that if $Q_{j} \neq \vec{A}_{1}^{n}, n \in \mathbb{N}, j>1$ then $T$ has unbounded growth on $Q_{j}$. We then have:

(1) $Q_{j}=w_{1}^{-}$

(2) $Q_{j}=w_{1}^{-} \vec{A}_{1}^{n}, n \in \mathbb{N}$

(3) $Q_{j}=\vec{A}_{1}^{-} \vec{A}_{1}^{n}, n \in \mathbb{N}$

(4) $Q_{j}=X_{j} V_{j} w_{1} Y_{j} W_{j} \quad X_{j} \in\left\{\epsilon, w_{1}^{-}, \vec{B}_{2}^{-}, \vec{A}_{1}^{-}\right\}, V_{j} \in\left\{\vec{A}_{1}\right\}^{*}, Y_{j} \in\left\{\epsilon, w_{1}, \vec{B}_{2}, \vec{A}_{1}\right\}, W_{j} \in\left\{w_{1}, \vec{B}_{2}, \vec{A}_{1}\right\}^{*}$

If $Q_{j}$ is one of the words of the forms given in (1), (2) or (3) then it can be easily checked that the word $Q_{j+1}$ produced after one round on $Q_{j}$ is a word of the form given in (4) and $l_{Q_{j+1}}>l_{Q_{j}}$. We thus only need to consider words $Q_{j}$ that are words of form (4).

It easily follows that $T$ must have unbounded growth on any word of form (4). Note first of all that none of the subwords in $X_{j}, V_{j}$ and $W_{j}$ can become shorter. This follows from Table 15.

Now, if $Y_{j}=w_{1}$ or $Y_{j}=\vec{B}_{2}$ we immediately have that $l_{Q_{j+1}}>l_{Q_{j}}$ since whatever shift $w_{1} Y_{j}$ is then entered with, it results in the production of a subword in $Q_{j+1}$ that is lengthier than $w_{1} Y_{j}$ (see Table 15). Furthermore $Q_{j+1}$ is again a word of the form (4).

If $Y_{j}=\vec{A}_{1}$ and $w_{1} \vec{A}_{1}$ is entered with shift $0 T$ produces $\vec{A}_{1} w_{1}^{2}$ in $Q_{j+1}$ from $w_{1} \vec{A}_{1}$ in $Q_{j}$. We thus again have that $l_{Q_{j+1}}>l_{Q_{j}}$ and $Q_{j+1}$ is again a word of form (4). If, on the other hand, $w_{1} \vec{A}_{1}$ is entered with shift 1 then $T$ reproduces $w_{1} \vec{A}_{1}$ in $Q_{j+1}$. However, if $w_{1} \vec{A}_{1}$ in $Q_{j}$ is entered with shift 1 then it must be 
the case that either $X_{j}=\vec{B}_{2}^{-}$or $X_{j}=\vec{A}_{1}^{-}$since $l_{w_{1}^{-}}$and $l_{\vec{A}_{1}}$ are even and we have assumed that $w_{1} \vec{A}_{1}$ is entered with shift 1 . But then it must be the case that $l_{Q_{j+1}}>l_{Q_{j}}$ since after one round on $Q_{j}, \vec{B}_{2}^{-}$in $Q_{j}$ results in $w_{1} \vec{A}_{1}$ (or $w_{1}^{-} \vec{A}_{1}$ ) and $\vec{A}_{1}^{-}$results in $w_{1}^{2}$ (or $w_{1}^{-} w_{1}$ ). Since $l_{w_{1}^{-} \vec{A}_{1}}>l_{\vec{B}_{2}^{-}}$and $l_{w_{1}^{-} w_{1}}>l_{\vec{A}_{1}^{-}}$, we again have that $l_{Q_{j+1}}>l_{Q_{j}}$ and $Q_{j+1}$ is again a word of form (4).

Finally, if $Y_{j}=\epsilon$ and $w_{1} Y_{j}$ is entered with shift 0 then $T$ produces $\overrightarrow{A_{1}}$ from $w_{1}$ in $Q_{j}$, when entered with shift $1, T$ produces $w_{1}$ in $Q_{j+1}$ from $w_{1}$ in $Q_{j}$. If $w_{1} Y_{j}$ is entered with shift 0 this either means that $Q_{j}=w_{1}, Q_{j}=\vec{A}_{1}^{n} w_{1}$ or $Q_{j}=w_{1}^{-} \vec{A}_{1}^{n} w_{1}$. In the first case it follows that $Q_{j+1}=\vec{A}_{1}^{-}$and thus $Q_{j+2}=w_{1}^{-} w_{1}$. We then have that $l_{Q_{j+2}}>l_{Q_{j}}$ and $Q_{j+2}$ is again a word of form (4). In the second case, if $Q_{j}=\vec{A}_{1}^{n} w_{1}$ then it easily follows that $Q_{j+1}=\vec{A}_{1}^{-} \vec{A}_{1}^{n}$ and thus $Q_{j+2}=\vec{B}_{2}^{-} \vec{B}_{2}^{n}$ and, since $l_{\vec{B}_{2}}>l_{\vec{A}_{1}}$ that $l_{Q_{j+2}}>l_{Q_{j}}$ and $Q_{j+2}$ is again a word of form (4). If $Q_{j}=w_{1}^{-} \vec{A}_{1}^{n} w_{1}$ then $Q_{j+1}=w_{1}^{-} \vec{A}_{1}^{n+1}$ but then $Q_{j+2}=w_{1} \vec{A}^{n+1}$ and thus $Q_{j+3}=\vec{A}_{1}^{-} \vec{B}_{2}^{n+1}$. Clearly, $l_{Q_{j+3}}>l_{Q_{j}}$ and $Q_{j+3}$ is again a word of form (4).

If $Y_{j}=\epsilon$ and $w_{1} Y_{j}$ in $Q_{j}$ is entered with shift 1 , then $T$ reproduces $w_{1} Y_{j}$ in $Q_{j+1}$. However, then we again have that either $X_{j}=\vec{B}_{2}^{-}$or $X_{j}=\vec{A}_{1}^{-}$and thus that we still have that $l_{Q_{j+1}}>l_{Q_{j}}$ and $Q_{j+1}$ is again a word of form (4).

It now follows that $T$ always has unbounded growth on any word $A_{0} \neq 0$ for which the word $Q_{2}$ produced after two rounds on $A_{0}$ is not a word $\vec{A}_{1}^{n}, n \in \mathbb{N}$.

Case II.C.1.d. $w_{1}=011\left(l_{w_{1}}=3, \# 1>2, w_{0}=1\right)$.

The proof of this case is very similar to the proof of Case II.C.1.c. The reason for this is that $w_{1}=011$ is the mirror image of $w_{1}=110$. As a consequence the words that can be produced through the table method from $w_{1}$ are symmetrical to the words produced from $w_{1}$ of Case II.C.1.c. through the table method.

Case II.C.2. $l_{w_{1}}>3\left(w_{0}=1, \# 1>2\right)$.

We split the case into two cases, determined by the parity of the number of 0 symbols between consecutive 1 symbols, i.e., $w_{1}=0^{r_{1}} 10^{\dot{x}_{1}} 10^{\dot{x}_{2}} 10^{\dot{x}_{3}} \ldots 0^{\dot{x}_{\# 1-1}} 10^{t_{1}}$, with any $\dot{x}_{i}, 0<i<\# 1$, odd and $w_{1}=0^{r_{1}} 10^{x_{1}} 10^{x_{2}} \ldots 0^{x_{1-1}} 10^{t_{1}}$, with at least one $x_{i}, 0<i<\# 1$ even.

Case II.C.2.a. $w_{1}=0^{r_{1}} 10^{\dot{x_{1}}} 10^{\dot{x_{2}}} 10^{\dot{x_{3}}} \ldots 0^{\dot{x}_{\# 1-1}} 10^{t_{1}}\left(w_{0}=1, \# 1>2, l_{w_{1}}>3\right)$.

Note that since all 1 symbols in $w_{1}$ are separated by an odd number of 0 symbols, $T$ will either read all 1 symbols in $w_{1}$ or no 1 in $w_{1}$ during one round on $w_{1}$. We split the case into two cases: $l_{w_{1}}=4$ and $l_{w_{1}}>4$.

Case II.C.2.a.1. $l_{w_{1}}=4\left(w_{0}=1, \# 1>2\right)$.

There are two possible tag systems in this class: either $w_{1}=1010$, or $w_{1}=0101$. It is easily proven for both tag systems that they either become periodic or have unbounded growth on any word $A_{0} \neq 0$. We will only consider the case with $w_{1}=0101$ as the other case is symmetrical to this case.

First, note that after one 0 -round on $w_{1} T$ produces $\vec{B}_{1}=11$, after one 1 -round, $T$ produces $w_{1}^{2}$. It easily follows that for any word $Q_{j}, j>1$ produced after $j$ rounds on $A_{0}$ we have:

$$
Q_{j}=X_{j} V_{j} \quad X_{j} \in\left\{\epsilon, \vec{B}_{1}^{-}, w_{1}^{-}\right\}, V_{j} \in\left\{\vec{B}_{1}, w_{1}\right\}^{*}
$$


Now, since $l_{w_{1}}$ and $\vec{B}_{1}$ are even it easily follows that if $X_{j}=\epsilon$ then $T$ is periodic on $Q_{j}$. It also follows that if $X_{j} \neq \epsilon$ then $T$ has unbounded growth on $Q_{j}$. Indeed, if $X_{j} \in\left\{\vec{B}_{1}^{-}, w_{1}^{-}\right\}$then every word $w_{1}$ in $Q_{j}$ produces $w_{1}^{2}$ in $Q_{j+1}$ and every word $\vec{B}_{1}$ produces $w_{1}$ in $Q_{j+1}$ and thus we have that $l_{Q_{j+1}}>l_{Q_{j}}$. Furthermore, since $l_{Q_{j}}$ is odd, we again have that $X_{j+1} \in\left\{\vec{B}_{1}^{-}, w_{1}^{-}\right\}$and thus $l_{Q_{j+1}}$ is odd.

Case II.C.2.a.2. $l_{w_{1}}>4\left(w_{0}=1, \# 1>2\right.$.) It is easily proven that any tag system $T$ in this class will always have unbounded growth on any initial word $A_{0} \neq 0$. This follows from Cases II.B. $i, i>2$ where it was proven that any tag system with $l_{w_{1}}>4, \# 1=2$ always has unbounded growth on any initial word $A_{0} \neq 0$ except for the tag systems with $w_{1}=01000$ and $w_{1}=00010$ for which there are two different periodic words. However, the only reason why these two tag systems can be periodic is that the word $\vec{A}_{1}$ contains only one word $w_{1}$. Clearly, this is impossible for the tag systems covered by Case II.C.2.a.2. Indeed, if $\vec{B}_{1}=111$ for this case then $\overrightarrow{A_{1}}$ must contain at least two words $w_{1}$.

Case II.C.2.b. $w_{1}=0^{r_{1}} 10^{x_{1}} 10^{x_{2}} 10^{x_{3}} \ldots 0^{x_{\# 1-1}} 10^{t_{1}}$, with at least one $x_{i}$ even $\left(w_{0}=1, \# 1>2, l_{w_{1}}>\right.$ 3).

It is easily proven that any tag system from this class always has unbounded growth on any word $A_{0} \neq 0$. First of all, it is trivial to prove that it takes at most two rounds of $T$ on $A_{0}$ to produce a word $Q_{j}$ that contains at least one word $w_{1}$. Now, depending on the shift $w_{1}$ is entered with, either at least two 1 symbols are read (thus resulting in the production of two words $w_{1}$ ) or at least one symbol 1 is read. If only one 1 is read this must result in the production of one word $w_{1}$ and at least one word $w_{0}$ (since $l_{w_{1}}>3$ ). In other words, whatever shift $w_{1}$ is entered with it always results in the production of a word that is lengthier than $l_{w_{1}}$ and that word again contains $w_{1}$. Hence the result of unbounded growth.

Case III. $w_{0}=0$.

As explained in Sec. 2.2, the only parameters used to determine the several subcases of Case III are parameter 1, the total number of 1 symbols \# 1 in the two appendants, and parameter 4, the parity of the number of 0 symbols separating consecutive 1 symbols in $w_{1}$. An important feature of tag systems $T$ of this case is the changes in the parity of the number of 0 symbols separating consecutive 1 symbols in $w_{1}$ with every new $s$-round on $w_{1}$. To see this, take for example the tag system with $w_{1}=101010100$. After one 0 -round on $w_{1} T$ produces the word $\vec{A}_{1}=w_{1} w_{1} w_{1} w_{1} 0$, after one 1-round $T$ produces 000 . Now, since the number of 0 symbols between the rightmost 1 in the first, second and third $w_{1}$ in $\overrightarrow{A_{1}}$ and the leftmost 1 in the second, third and last $w_{1}$ in $\overrightarrow{A_{1}}$, respectively, is even, it immediately follows that whatever shift $\overrightarrow{A_{1}}$ is entered with it results in the production of a word that contains $\vec{A}_{1}$ twice. This implies that this tag system always has unbounded growth on any initial word $A_{0}$ that results in the production of a word that contains $\overrightarrow{A_{1}}$ at least once. If, on the other hand, $w_{1}=10101010$ then this tag system will either halt or have unbounded growth. The reason for this is that the distance between consecutive 1 symbols in the word produced after one 0 -round on $w_{1}$ will always remain odd.

In the remaining sections any sequence of 0 symbols $0^{i}$ will be represented as $i$ to avoid complicated notations. For any tag system $T$ with $\# 1>0$ the word $w_{1}$ will be represented as $w_{1}=$ $t_{1} 1 x_{1} 1 x_{2} 1 \ldots x_{\# 1-1} 1 r_{1}$ where $r_{1}$ and $t_{1}$ denote the number of 0 symbols to the left-hand side of the leftmost 1 in $w_{1}$ and to the right-hand side of the rightmost 1 in $w_{1}$, respectively; $x_{i}$ stands for the sequence of 0 symbols separating consecutive 1 symbols in $w_{1}$. Indexed variables $k_{i}$ represent some sequence of 0 symbols of length $k_{i}$. 
Let $T$ be a tag system with $\# 1>0, \vec{A}_{0}=w_{1}$. Since $\# 1>0$ it must be the case that after $n \in \mathbb{N}$ $s$-rounds on $w_{1}$ at least one word $\overrightarrow{A_{n}}$ is produced that again contains at least one word $w_{1}$. In what follows any such word $\overrightarrow{A_{n}}$ will be represented as:

$$
\vec{A}_{n}=\left[X_{1} / 2^{n}\right]\left[X_{2} / 2^{n-1}\right] \ldots\left[X_{n-1} / 4\right]\left[X_{n} / 2\right] w_{1} k_{n_{1}} w_{1} k_{n_{2}} \ldots k_{n_{j}} w_{1}\left[Y_{n} / 2\right]\left[Y_{n-1} / 4\right] \ldots\left[Y_{2} / 2^{n-1}\right]\left[Y_{1} / 2^{n}\right]
$$

Now, let:

$$
\overrightarrow{A_{i}}=\left[X_{1} / 2^{i}\right]\left[X_{2} / 2^{i-1}\right] \ldots\left[X_{i-1} / 4\right]\left[X_{i} / 2\right] w_{1} k_{i_{1}} w_{1} k_{i_{2}} \ldots k_{i_{j}} w_{1}\left[Y_{n} / 2\right]\left[Y_{i-1} / 4\right] \ldots\left[Y_{2} / 2^{i-1}\right]\left[Y_{1} / 2^{i}\right]
$$

be some word from which $\vec{A}_{n}$ is produced after $n s$-rounds with $\vec{A}_{0}=w_{1}$. Then each $X_{i}$ in $\vec{A}_{n}$ represents the total number of 0 and 1 symbols erased in $w_{1} k_{i_{1}} w_{1} k_{i_{2}} \ldots k_{i_{j}} w_{1}$ to the left of the leftmost 1 read in $\vec{A}_{i-1}$, and each $Y_{i}$ in $\vec{A}_{n}$ represents the total number of 0 and 1 symbols erased to the right of the rightmost 1 that is read in $\vec{A}_{i-1}$. For example, if $n=1$ then $\vec{A}_{1}=\left[X_{1} / 2\right] w_{1} k_{1_{1}} w_{1} k_{1_{2}} \ldots k_{1_{j}} w_{1}\left[Y_{1} / 2\right]$ and $X_{1}$ is the number of 0 symbols produced from the sequence of 0 symbols preceding the first $1 \mathrm{read}$ in $w_{1}$ from which $T$ produces the leftmost $w_{1}$ in $\vec{A}_{1}$ and $Y_{1}$ is the number of 0 symbols produced from the sequence of 0 symbols that follows the last 1 read in $w_{1}$ from which $T$ produces the leftmost $w_{1}$ in $\overrightarrow{A_{1}}$.

Before we begin to give the subcases for Case III we will prove that there is an $n$ such that for any number $i \in \mathbb{N}$ the sequences $\left[X_{1} / 2^{n+i}\right]\left[X_{2} / 2^{n+i-1}\right] \ldots\left[X_{n+i} / 2\right]$ and $\left[Y_{n+i} / 2\right] \ldots\left[Y_{2} / 2^{n+i-1}\right]\left[Y_{1} / 2^{n+i}\right]$ in $\vec{A}_{n+i}$ are bounded. It is this property that largely determines the ultimate behavior for any tag system $T$ with $\mu=v=2, w_{0}=0$ for some initial word $A_{0}$. This property is proven through Lemma's 3 and 4 . Lemma 3 proves that one can determine values $X_{\max }, Y_{\max }, X_{\min }, Y_{\min }$ such that for every $X_{i}$ and $Y_{i}$, $X_{i}=X_{\max }$ or $X_{i}=X_{\min }$ and $Y_{i}=Y_{\max }$ or $Y_{i}=Y_{\min }$. This Lemma is used to prove Lemma 4. This Lemma proves the above boundedness property.

Lemma 3. Given a 2-tag system $T$ with $\mu=v=2, w_{0}=0$ and $\# 1>0, w_{1}=t_{1} 1 x_{1} 1 x_{2} 1 \ldots 1 r_{1}$, then one can determine values $X_{\max }, Y_{\max }, X_{\min }, Y_{\min }$ for any word $\overrightarrow{A_{n}}$ :

$$
\vec{A}_{n}=\left[X_{1} / 2^{n}\right]\left[X_{2} / 2^{n-1}\right] \ldots\left[X_{n-1} / 4\right]\left[X_{n} / 2\right] w_{1} k_{n_{1}} w_{1} k_{n_{2}} \ldots k_{n_{j}} w_{1}\left[Y_{n} / 2\right]\left[Y_{n-1} / 4\right] \ldots\left[Y_{2} / 2^{n-1}\right]\left[Y_{1} / 2^{n}\right]
$$

produced after $n s$-rounds on $w_{1}$ that contains at least one word $w_{1}$, for every $X_{i}, Y_{i} \in \vec{A}_{n}, X_{i}=X_{\max }$ or $X_{i}=X_{\min }$ and $Y_{i}=Y_{\max }$ or $Y_{i}=Y_{\min }$.

\section{Proof:}

In order to prove the Lemma we split Case III into three global subcases, determined by the parities of the sequences of 0 symbols between consecutive 1 symbols in $w_{1}$, i.e., $w_{1}=r_{1} 1 t_{1}(\# 1=1), w_{1}=$ $r_{1} 1 x_{1} 1 x_{2} 1 x_{3} \ldots x_{\# 1-1} 1 t_{1}, \# 1 \geq 2$, with at least one $x_{i}$ even, and $w_{1}=r_{1} 1 \dot{x}_{1} 1 \dot{x}_{2} 1 \dot{x}_{3} \ldots \dot{x}_{\# 1-1} 1 t_{1}, \# 1 \geq$ 2 , with every $\dot{x}_{i}$ odd.

Case a. $w_{1}=r_{1} 1 t_{1}, \# 1=1$.

After one $s$-round on $w_{1} T$ either produces the word $\vec{A}_{1}=\left[X_{1} / 2\right] w_{1}\left[Y_{1} / 2\right]$, with $X_{1}=r_{1}, Y_{1}=t_{1}$ or a sequence of 0 symbols. After one $s$-round of $T$ on $\vec{A}_{1} T$ again produces either a sequence of 0 symbols or the word $\overrightarrow{A_{2}}=\left[X_{1} / 4\right]\left[X_{2} / 2\right] w_{1}\left[Y_{2} / 2\right]\left[Y_{1} / 4\right], X_{2}=r_{1}, Y_{2}=t_{1}$. It easily follows from this that after $n s$-rounds of $T$ on $w_{1} T$ produces either a sequence of 0 symbols or a word 
$\vec{A}_{n}=\left[X_{1} / 2^{n}\right]\left[X_{2} / 2^{n-1}\right] \ldots\left[X_{n-1} / 4\right]\left[X_{n} / 2\right] w_{1}\left[Y_{n} / 2\right]\left[Y_{n-1} / 4\right] \ldots\left[Y_{2} / 2^{n-1}\right]\left[Y_{1} / 2^{n}\right]$ since any word $\vec{A}_{n}$ contains only one word $w_{1}$. It follows that for any such word $\vec{A}_{n}$ that contains at least one word $w_{1}$ it must be the case that each $X_{i}=r_{1}, Y_{i}=t_{1}$ and thus we have that each $X_{i}=X_{\max }=X_{\min }=r_{1}$ and $Y_{i}=Y_{\max }=Y_{\min }=t_{1}$.

Case b. $w_{1}=r_{1} 1 x_{1} 1 x_{2} 1 x_{3} \ldots x_{p_{1}} 1 t_{1},(\# 1 \geq 2)$, with at least one $x_{i}$ even.

Note that for any tag system $T$ for which $w_{1}$ that contains at least two 1 symbols separated by an even number of 0 symbols, $T$ always reads at least one 1 in $w_{1}$ during one $s$-round on $w_{1}$. Let:

$$
\vec{A}_{n}=\left[X_{1} / 2^{n}\right]\left[X_{2} / 2^{n-1}\right] \ldots\left[X_{n-1} / 4\right]\left[X_{n} / 2\right] w_{1} k_{n_{1}} w_{1} k_{n_{2}} \ldots k_{n_{j}} w_{1}\left[Y_{n} / 2\right]\left[Y_{n-1} / 4\right] \ldots\left[Y_{2} / 2^{n-1}\right]\left[Y_{1} / 2^{n}\right]
$$

be some word produced after $n s$-rounds on $w_{1}$ and let:

$$
\overrightarrow{A_{i}}=\left[X_{1} / 2^{i}\right]\left[X_{2} / 2^{i-1}\right] \ldots\left[X_{i-1} / 4\right]\left[X_{i} / 2\right] w_{1} k_{i_{1}} w_{1} k_{i_{2}} \ldots k_{i_{j}} w_{1}\left[Y_{n} / 2\right]\left[Y_{i-1} / 4\right] \ldots\left[Y_{2} / 2^{i-1}\right]\left[Y_{1} / 2^{i}\right]
$$

be any of the words from which $\vec{A}_{n}$ is produced after $n-i s$-rounds on $\vec{A}_{i}$ with $\vec{A}_{0}=w_{1}$. It then easily follows that for each $X_{i}$ in $\vec{A}_{n}$ either $X_{i}=t_{1}$ (when the first 1 read in $\vec{A}_{i}$ from which $T$ produces the leftmost $w_{1}$ in $\vec{A}_{i+1}$ is the leftmost 1 of the leftmost word $w_{1}$ in $\vec{A}_{i}$ ) or $X_{i}=t_{1}+\dot{x}_{1}+\dot{x}_{2}+\ldots+\ddot{x}_{j}+j$ with $\ddot{x}_{j}$ the first sequence of 0 symbols in $w_{1}$ that has even length (when the first 1 read in $\vec{A}_{i}$ from which $T$ produces the leftmost $w_{1}$ in $\vec{A}_{i+1}$ is not the leftmost 1 in the leftmost word $w_{1}$ in $\overrightarrow{A_{i}}$ ). Similarly, for each $Y_{i}$ in $\vec{A}_{n}$, either $Y_{i}=t_{1}$ or $Y_{i}=\ddot{x}_{j^{\prime}}+\dot{x}_{j^{\prime}+1}+\ldots .+\dot{x}_{\# 1-1}+r_{1}+\# 1-j^{\prime}$ where $j^{\prime}$ is the index of the last sequence of 0 symbols in $w_{1}$ that has even length. We then have that $X_{\max }=t_{1}+\dot{x}_{1}+\dot{x}_{2}+\ldots+\ddot{x}_{j}+j, X_{\min }=r_{1}$ and $Y_{\min }=t_{1}, Y_{\max }=\ddot{\dot{x}_{j^{\prime}}}+\dot{x}_{j^{\prime}+1}+\ldots .+\dot{x}_{\# 1-1}+r_{1}+\# 1-j^{\prime}$ and for any $X_{i}, Y_{i}$ in $\vec{A}_{n}, X_{i}=X_{\max }$ or $X_{i}=X_{\min }$ and $Y_{i}=Y_{\max }$ or $Y_{i}=Y_{\min }$.

Case c. $w_{1}=r_{1} 1 \dot{x}_{1} 1 \dot{x}_{2} 1 \dot{x}_{3} \ldots \dot{x}_{p_{1}} 1 t_{1},(\# 1 \geq 2)$, with every $\dot{x}_{i}$ odd.

Since all 1 symbols in $w_{1}$ are separated by an odd number of 0 symbols, $T$ will either produce a sequence of 0 symbols or the following word after one $s$-round of $T$ on $w_{1}$ :

$$
\overrightarrow{A_{1}}=\left[X_{1} / 2\right] w_{1}\left[\dot{x}_{1} / 2\right] w_{1}\left[\dot{x}_{2} / 2\right] w_{1} \ldots w_{1}\left[\dot{x}_{\# 1-1} / 2\right] w_{1}\left[Y_{1} / 2\right]
$$

with $X_{1}=r_{1}, Y_{1}=t_{1}$.

Now, if there is at least one $j$ such that $t_{1}+\left[\dot{x}_{j} / 2\right]+r_{1}$ is even, where $t_{1}+\left[\dot{x}_{j} / 2\right]+r_{1}$ gives the total number of 0 symbols separating the rightmost 1 symbol in a word $w_{1}$ from the leftmost 1 symbol in the next word $w_{1}$ in $w_{1}\left[\dot{x}_{1} / 2\right] w_{1}$, then the case reduces to case $\mathrm{b}$ since then there is always at least one $w_{1}$ for which the total number of 1 symbols in $w_{1}$ will be read by $T$. It then immediately follows that $X_{\min }=r_{1}, X_{\max }=j l_{w_{1}}+\left[\dot{x}_{1} / 2\right]+\left[\dot{x}_{2} / 2\right]+\ldots+\left[\dot{x}_{j} / 2\right]+r_{1}, Y_{\min }=t_{1}, Y_{\max }=r_{1}+\left[\dot{x}_{j^{\prime}} / 2\right]+$ $\left[x_{j^{\prime}+1} / 2\right]+\ldots+\left[\dot{x}_{\# 1-1} / 2\right]+\left(\# 1-j^{\prime}\right) l_{w_{1}}$ with $t_{1}+\left[\dot{x}_{j} / 2\right]+r_{1}$ and $t_{1}+\left[\dot{x_{j^{\prime}}} / 2\right]+t_{1}$ respectively the leftmost and rightmost sequence of 0 symbols in $\vec{A}_{1}$ that is even. For any word $\vec{A}_{n}$ produced after $n s$ rounds on $w_{1}$ and any $X_{i}$ and $Y_{i}$ in $\vec{A}_{n}$ produced after $n-1 s$-rounds on $\overrightarrow{A_{1}}, X_{i}=X_{\max }$ or $X_{i}=X_{\min }$ and $Y_{i}=Y_{\max }$ or $Y_{i}=Y_{\min }$.

If there is no such $j$ then $T$ produces either a sequence of 0 symbols or the following word:

$$
\overrightarrow{A_{2}}=\left[X_{1} / 4\right] \vec{A}_{1}\left[\dot{x}_{1} / 4\right] \vec{A}_{1}\left[\dot{x}_{2} / 4\right] \vec{A}_{1} \ldots \vec{A}_{1}\left[\dot{x}_{\# 1-1} / 4\right] \vec{A}_{1}\left[Y_{1} / 4\right]
$$

after one $s$-round on $\vec{A}_{1}$. Again, if there is at least one $j$ such that $t_{1}+\left[Y_{1} / 2\right]+\left[\dot{x}_{j} / 4\right]+\left[X_{1} / 2\right]+r_{1}$ is even the case reduces to case $\mathrm{b}$, and we can again determine $X_{\max }, X_{\min }, Y_{\max }$ and $Y_{\min }$. 
If there is no such $j$ then $T$ will again produce a sequence of 0 symbols or a word $\vec{A}_{3}$ after one $s$-round on $\vec{A}_{2}, \ldots$

Generally speaking, for any $T$ from this class, there are two possibilities. The first is that there is an $n$ such that the word:

$$
\vec{A}_{n}=\left[X_{1} / 2^{n}\right] \vec{A}_{n-1}\left[\dot{x}_{1} / 2^{n}\right] \vec{A}_{n-1}\left[\dot{x}_{2} / 2^{n}\right] \vec{A}_{n-1} \ldots \vec{A}_{n-1}\left[\dot{x}_{\# 1-1} / 2^{n}\right] \vec{A}_{n-1}\left[Y_{1} / 2^{n}\right]
$$

contains at least one sequence of 0 symbols $t_{1}+\left[Y_{n-1} / 2\right]+\left[Y_{n-2} / 4\right]+\ldots\left[Y_{1} / 2^{n}\right]+\left[\dot{x}_{j} / 2^{n}\right]+\left[X_{1} / 2^{n}\right]+$ $\ldots+\left[X_{n-2} / 4\right]+\left[X_{n-1} / 2\right]+r_{1}$ that is even. The second possibility is that there exists no such $n$. If there exists such an $n$, then the case reduces to case $\mathrm{b}$ and $X_{\max }, X_{\min }, Y_{\max }$ and $Y_{\min }$ can be determined. If there exists no such $n$ then it immediately follows that for any $\vec{A}_{n}, X_{\max }=X_{\min }=r_{1}, Y_{\max }=$ $Y_{\min }=t_{1}$.

Lemma 4. Given a 2-tag system with $\mu=v=2, w_{0}=0$ and $\# 1>0, w_{1}=t_{1} 1 x_{1} 1 x_{2} 1 \ldots 1 r_{1}$, then it is possible to determine values $n, \operatorname{Max}_{\mathbf{X}}, \operatorname{Max}_{\mathbf{Y}} \in \mathbb{N}$ such that for any word $\vec{A}_{n+i}, i \in \mathbb{N}$ :

$$
\vec{A}_{n+i}=\left[X_{1} / 2^{n+i}\right] \ldots\left[X_{n+i-1} / 4\right]\left[X_{n+i} / 2\right] w_{1} k_{n+i_{1}} w_{1} \ldots k_{n+i_{j}} w_{1}\left[Y_{n+i} / 2\right]\left[Y_{n+i-1} / 4\right] \ldots\left[Y_{1} / 2^{n+i}\right]
$$

produced after $n s$-rounds on $w_{1}$ that contains at least one word $w_{1}$ we have that

$$
\begin{gathered}
{\left[X_{1} / 2^{n+i}\right] \ldots\left[X_{n+i-1} / 4\right]\left[X_{n+i} / 2\right] \leq \operatorname{Max}_{\mathbf{X}}} \\
{\left[Y_{n+i} / 2\right]\left[Y_{n+i-1} / 4\right] \ldots\left[Y_{1} / 2^{n+i}\right] \leq \operatorname{Max}_{\mathbf{Y}}}
\end{gathered}
$$

\section{Proof:}

We will only prove that any sequence of 0 symbols $\left[X_{1} / 2^{n+i}\right] \ldots\left[X_{n+i-1} / 4\right]\left[X_{n+i} / 2\right]$ will become bounded by some constant $\operatorname{Max}_{\mathbf{X}}$ after a certain number $n$ of $s$-rounds of $T$ on $w_{1}$. The proof for the right-hand side is symmetrical to this case. ${ }^{3}$

Let $T$ be a 2-tag system with $\mu=v=2, w_{0}=0$ and $\# 1>0$, then for any word $\vec{A}_{m}$ produced after $m$ $s$-rounds on $w_{1}$ that contains at least one word $w_{1}$ :

$$
\vec{A}_{m}=\left[X_{1} / 2^{m}\right]\left[X_{2} / 2^{m-1}\right] \ldots\left[X_{m} / 2\right] w_{1} k_{m_{1}} w_{1} k_{m_{2}} \ldots k_{m_{j}} w_{1}\left[Y_{m} / 2\right] \ldots\left[Y_{2} / 2^{m-1}\right]\left[Y_{1} / 2^{m}\right]
$$

It follows from Lemma 3 that for each $X_{i}$ and $Y_{i}$ in $\vec{A}_{m}, X_{i} \leq X_{\max }, Y_{i} \leq Y_{\max }$. Note also that any $\left[X_{i} / 2^{m-i+1}\right]$ is in fact $\left\lfloor X_{i} / 2^{m-i+1}\right\rfloor$ or $\left\lceil X_{i} / 2^{m-i+1}\right\rceil$. In the first case, $\left[X_{i+1} / 2^{m-i+2}\right]$ is in fact $\left\lceil X_{i+1} / 2^{m-i+2}\right\rceil$, in the second, $\left[X_{i+1} / 2^{m-i+2}\right]$ is in fact $\left\lfloor X_{i+1} / 2^{m-i+2}\right\rfloor$. The reason for this is that if one sequence of 0 symbols $\left[X_{i} / 2^{m-i+1}\right]$ is entered with shift 0 and $\left[X_{i} / 2^{m-i+1}\right]$ is odd, then the next sequence of 0 symbols will be entered with shift 1 , and vice versa.

We can now determine values $\operatorname{Max}_{\mathbf{X}}$ and $\operatorname{Max}_{\mathbf{Y}}$ and $n$. Let us assume the worst case such that each $X_{i}$ and $Y_{i}$ in $\vec{A}_{m}$ has the maximum value $X_{\max }$ and $Y_{\max }$, respectively. Evidently, there must be an $n$ such that either $\left\lceil X_{\max } / 2^{n}\right\rceil=1,\left\lfloor X_{\max } / 2^{n-1}\right\rfloor=0$ or $\left\lfloor X_{\max } / 2^{n}\right\rfloor=0,\left\lceil X_{\max } / 2^{n-1}\right\rceil=1$. Now, given the word:

$$
\vec{A}_{n}=\left[X_{\max } / 2^{n}\right]\left[X_{\max } / 2^{n-1}\right] \ldots\left[X_{\max } / 2\right] w_{1} k_{n_{1}} w_{1} k_{n_{2}} \ldots k_{n_{j}} w_{1}\left[Y_{\max } / 2\right] \ldots\left[Y_{\max } / 2^{n}\right]
$$

\footnotetext{
${ }^{3}$ I am indebted to an anonymous referee for pointing out a serious error in a previous version of this proof.
} 
Then, after one more $s$-round on $\vec{A}_{n} T$ produces:

$\vec{A}_{n+1}=\left[X_{\max } / 2^{n+1}\right]\left[X_{\max } / 2^{n}\right]\left[X_{\max } / 2^{n-1}\right] \ldots\left[X_{\max } / 2\right] w_{1} k_{n+1_{1}} \ldots k_{n+1_{j}} w_{1}\left[Y_{\max } / 2\right] \ldots\left[Y_{\max } / 2^{n+1}\right]$

Now, if $\left[X_{\max } / 2^{n}\right]=0$ in $\vec{A}_{n}$ then $\left[X_{\max } / 2^{n+1}\right]=0$ in $\vec{A}_{n+1}$. If $\left[X_{\max } / 2^{n}\right]=1,\left[X_{\max } / 2^{n-1}\right]=0$ in $\overrightarrow{A_{n}}$, then $\left[X_{\max } / 2^{n}\right]=0$ in $\vec{A}_{n+1}$ and $\left[X_{\max } / 2^{n+1}\right]=\left[X_{\max } / 2^{n}\right]$. It thus immediately follows that:

$$
\vec{A}_{n+1}=\left[X_{\max } / 2^{n}\right]\left[X_{\max } / 2^{n-1}\right] \ldots\left[X_{\max } / 2\right] w_{1} k_{n+1_{1}} \ldots k_{n+1_{j}} w_{1}\left[Y_{\max } / 2\right] \ldots\left[Y_{\max } / 2^{n+1}\right]
$$

and thus that for any word $\vec{A}_{n+i}$ the sequence of 0 symbols $\left[X_{1} / 2^{n+i}\right]\left[X_{2} / 2^{n+i-1}\right] \ldots\left[X_{n+i-1} / 4\right]\left[X_{n+i} / 2\right], X_{i}=$ $X_{\min }$ or $X_{i}=X_{\max }$ is bounded either by:

$$
\begin{gathered}
\left\lfloor X_{\max } / 2^{n}\right\rfloor\left\lceil X_{\max } / 2^{n-1}\right\rceil \ldots\left[X_{\max } / 2\right] \\
\text { If }\left\lfloor X_{\max } / 2^{n}\right\rfloor\left\lceil X_{\max } / 2^{n-1}\right\rceil \ldots\left[X_{\max } / 2\right]>\left\lceil X_{\max } / 2^{n}\right\rceil\left\lfloor X_{\max } / 2^{n-1}\right\rfloor \ldots\left[X_{\max } / 2\right] \\
\text { or by } \\
\left\lceil X_{\max } / 2^{n}\right\rceil\left\lfloor X_{\max } / 2^{n-1}\right\rfloor \ldots\left[X_{\max } / 2\right] \\
\text { If }\left\lceil X_{\max } / 2^{n}\right\rceil\left\lfloor X_{\max } / 2^{n-1}\right\rfloor \ldots\left[X_{\max } / 2\right]>\left\lfloor X_{\max } / 2^{n}\right\rfloor\left\lceil X_{\max } / 2^{n-1}\right\rceil \ldots\left[X_{\max } / 2\right]
\end{gathered}
$$

Case III.A. $\# 1=0\left(w_{0}=0\right)$.

It is trivial to prove that any tag system $T$ from this class will always halt on any initial word $A_{0}$. First of all, after one round of $T$ on $A_{0} T$ produces the word $Q_{1}=0^{n}, n \in \mathbb{N}$. Secondly, $T$ always halts on any sequence of 0 symbols because at each computation step for every 0 appended 2 are deleted.

Case III.B. $\# 1=1,\left(w_{0}=0\right)$.

Any tag systems $T$ with $w_{1}=r_{1} 1 t_{1}$ covered by this case will either halt or become periodic on any initial word $A_{0}$ because the table method halts for $T$. Note that this is case a of the proof of Lemma 3 and thus we have that after $n s$-rounds of $T$ on $w_{1} T$ produces either a sequence of 0 symbols or a word:

$$
\vec{A}_{n}=\left[r_{1} / 2^{i}\right]\left[r_{1} / 2^{i-1}\right] \ldots\left[r_{1} / 2\right] w_{1}\left[t_{1} / 2\right] \ldots\left[t_{1} / 2^{n-1}\right]\left[t_{1} / 2^{n}\right]
$$

since $w_{1}$ contains only one symbol 1 . Now, it follows from Lemma 4 that we can determine an $n$ such that for any word $\vec{A}_{n+i}$ produced after $n+i s$-rounds on $w_{1}$ the sequence of 0 symbols to the left-hand of the leftmost and right-hand side of the rightmost 1 in $w_{1}$ in $\vec{A}_{n+i}$ is bounded by come constant. This implies that there is thus but a finite number of different words $\vec{A}_{i}$ that can be produced with the table method and thus the table method halts for $T$.

Case III.C. $\# 1=2,\left(w_{0}=0\right)$.

We split the case into two subcases, determined by the parity of the number of 0 symbols between the two 1 symbols in $w_{1}$, i.e., $w_{1}=r_{1} 1 \ddot{x}_{1} 1 t_{1}$ or $w_{1}=r_{1} 1 \dot{x_{1}} 1 t_{1}$.

Case III.C.1. $w_{1}=r_{1} 1 \ddot{x}_{1} 1 t_{1}\left(w_{0}=0, \# 1=2\right)$.

Note that after one $s$-round of $T$ on $w_{1}, T$ always produces one $w_{1}$, surrounded by a finite number of 0 symbols and thus this case is similar to Case III.B. I.e., only a finite number of words can be produced 
by the table method when applied to $w_{1}$ and it thus follows that $T$ always halts or becomes periodic on any initial word $A_{0}$.

Case III.C.2. $w_{1}=r_{1} 1 \dot{x_{1}} 1 t_{1}\left(w_{0}=0, \# 1=2\right)$

For any tag system $T$ covered by this case, either $T$ only reads 0 symbols or two 1 symbols during an $s$-round on $w_{1}$. Thus, after one $s$-round on $w_{1} T$ either produces a sequence of 0 symbols, ultimately leading to the production of $\epsilon$, or the following word:

$$
\overrightarrow{A_{1}}=\left[r_{1} / 2\right] w_{1}\left[\dot{x_{1}} / 2\right] w_{1}\left[t_{1} / 2\right]
$$

The parity of the length of $t_{1}\left[\dot{x_{1}} / 2\right] r_{1}$ plays a significant role in the ultimate behavior of $T$. Indeed, if $t_{1}\left[\dot{x_{1}} / 2\right] r_{1}$ is odd then both words $w_{1}$ in $\overrightarrow{A_{1}}$ will be entered with the same shift. If $t_{1}\left[\dot{x_{1}} / 2\right] r_{1}$ is even and the first $w_{1}$ in $\overrightarrow{A_{1}}$ is entered with shift 0 then the second $w_{1}$ will be entered with shift 1 and vice versa.

Then, if $t_{1}\left[\dot{x_{1}} / 2\right] r_{1}$ in $\vec{A}_{1}$ has even length $T$ produces one of the following words:

$$
k_{1,1} \vec{A}_{1} k_{1,2}
$$

or:

$$
k_{1,3} \vec{A}_{1} k_{1,4}
$$

after one $s$-round on $\vec{A}_{1}$, where $k_{i, j}$ indicates some sequence of 0 symbols. It then easily follows that if $t_{1}\left[\dot{x_{1}} / 2\right] r_{1}$ in $\overrightarrow{A_{1}}$ has even length, then $T$ will either become periodic or halt on $A_{0}$ due to the boundedness property on the number of 0 symbols the left and right of any word $\vec{A}_{n}$ produced after $n-1 s$-rounds on $\overrightarrow{A_{1}}$ (Lemma 4).

If $t_{1}\left[\dot{x}_{1} / 2\right] r_{1}$ has odd length, $T$ produces:

$$
\overrightarrow{A_{2}}=\left[r_{1} / 4\right] \overrightarrow{A_{1}}\left[\dot{x_{1}} / 4\right] \overrightarrow{A_{1}}\left[t_{1} / 4\right]
$$

after one $s$-round on $\vec{A}_{1}$ or a sequence of 0 symbols depending on the shift with which $\vec{A}_{1}$ is entered and the number of 0 symbols preceding the first 1 in $\overrightarrow{A_{1}}$.

The ultimate behavior of $T$ depends on the parity of the length of $t_{1}\left[t_{1} / 2\right]\left[\dot{x_{1}} / 4\right]\left[r_{1} / 2\right] r_{1}$ in $\overrightarrow{A_{2}}$. If $t_{1}\left[t_{1} / 2\right]\left[\dot{x_{1}} / 4\right]\left[r_{1} / 2\right] r_{1}$ has even length, $T$ produces either one of the following words after one $s$-round on $\overrightarrow{A_{2}}$ :

$$
k_{2,1} \vec{A}_{2} k_{2,2}
$$

or:

$$
k_{2,3} \vec{A}_{2} k_{2,4}
$$

It thus follows that if $t_{1}\left[t_{1} / 2\right]\left[\dot{x}_{1} / 4\right]\left[r_{1} / 2\right] r_{1}$ is even that $T$ will always either halt or become periodic on any initial word $A_{0}$.

If $t_{1}\left[t_{1} / 2\right]\left[\dot{x_{1}} / 4\right]\left[r_{1} / 2\right] r_{1}$ has odd length, then after one $s$-round on $\overrightarrow{A_{2}} T$ again either produces a sequence of 0 symbols or a word $\vec{A}_{3}$ containing two words $\vec{A}_{2}, \ldots$

It easily follows that tag systems $T$ from this class will always become periodic or halt on any word $A_{0}$ if there is an $n$ such that after $n s$-rounds of $T$ on $w_{1} T$ produces the word:

$$
\vec{A}_{n}=\left[r_{1} / 2^{n}\right] \vec{A}_{n-1}\left[\dot{x_{1}} / 2^{n}\right] \vec{A}_{n-1}\left[t_{1} / 2^{n}\right]
$$


with $\left.t_{1}\left[t_{1} / 2\right] \ldots\left[t_{1} / 2^{n}\right]\left[\left(\dot{x_{1}}-1\right) / 2^{n}\right]\left[r_{1} / 2^{n}\right] \ldots\left[r_{1} / 2\right]+r_{1}\right]$ of even length. It easily follows from Lemma 4 that we can determine (in a finite number of steps) for any $T$ covered by this case whether or not there exists such an $n$. If not, $T$ will either halt or have unbounded growth on $A_{0}$ since for any word $\vec{A}_{i}$ produced after $i s$-rounds on $w_{1} T$ either produces a sequence of 0 symbols or a new word $\vec{A}_{i+1}$ containing two words $\vec{A}_{i}$. In this last case it can be decided whether or not $T$ will have unbounded growth. The reason for this is that (1) one can determine an $m$ such that for any word $\vec{A}_{m+i}$ the length of the sequences of 0 symbols separating consecutive 1 symbols in $\vec{A}_{m+i}$ is bounded (Lemma 4) (2) for any sequence $Q_{j}$ produced after $j$ rounds on $A_{0}$ the length of the sequences of 0 symbols separating consecutive words $\vec{A}_{k}$ is bounded (3) given $m$ one can determine the parity of any word $\vec{A}_{k}$. Then, since any word $Q_{m+j}$ produced after $m+j$ rounds on $A_{0}$ contains only words $\vec{A}_{m+i}, i \in \mathbb{N}$ and sequences of 0 symbols, the result easily follows.

Case III.D. $\# 1 \geq 3,\left(w_{0}=0\right)$.

We split the case into two subcases, determined by the parity of the number of 0 symbols separating consecutive 1 symbols in $w_{1}$, i.e., $w_{1}=r_{1} 1 \dot{x}_{1} 1 \dot{x}_{2} 1 \ldots 1 \dot{x}_{\# 1-1} 1 t_{1}$ with every $\dot{x}_{i}$ odd or $w_{1}=$ $r_{1} 1 x_{1} 1 x_{2} 1 \ldots x_{\# 1-1} 1 t_{1}$ with at least one $x_{i}$ even. Note that the fundamental difference between these two cases is the fact that with all 1 symbols in $w_{1}$ separated by an odd number of 0 symbols either all 1 symbols or no 1 symbols are read during one $s$-round on $w_{1}$. If at least two 1 symbols are separated by an even number of 0 symbols, either at least one 1 or two 1 symbols will be read by $T$ during one $s$-round on $w_{1}$.

Case III.D.1. $w_{1}=r_{1} 1 x_{1} 1 x_{2} 1 \ldots x_{\# 1-1} 1 t_{1}\left(\# 1 \geq 3, w_{0}=0\right)$

Clearly, for any of these tag systems either at least one 1 or at least two 1 symbols are read during one $s$-round on $w_{1}$. This means that none of these tag systems can halt on an initial word $A_{0}$ in which $T$ reads at least one 1.

Now, for any tag system $T$ with $\# 1>3$, if $T$ reads at least two 1 symbols in $w_{1}$ whatever shift $w_{1}$ is entered with, then $T$ always produces $w_{1}^{n}, n \geq 2$ after one $s$-round on $w_{1}$. From this it easily follows that $T$ always has unbounded growth on any initial word $A_{0}$ for which $T$ reads at least one 1 during one round on $A_{0}$. If no 1 is read in $A_{0}$ then $T$ will halt on $A_{0}$.

If $T$ always either reads one 1 or at least two 1 symbols during one $s$-round on $w_{1}$, then it follows that there is either one sequence $x_{i}$ of 0 symbols in $w_{1}$ that is even or there are two consecutive sequences $x_{i}, x_{i+1}$ that are even. If there is only one $x_{i}$ that is even then either $i=1$ or $i=\# 1-1$. Otherwise, if we have a pair of $\left(x_{i}, x_{i+1}\right)$ that are even then $i \in\{1, \ldots, \# 1-1\}$. For the sake of simplicity we assume that there is only one sequence of 0 symbols $x_{i}$ that is even and that $i=\# 1-1$, thus $w_{1}=$ $r_{1} 1 \dot{x}_{1} 1 \dot{x}_{2} \ldots 1 \ddot{x}_{\# 1-1} 1 r_{1}$. The other cases easily reduce to this case. After one $s$-round on $w_{1} T$ produces either one of the following words:

$$
\begin{gathered}
\vec{A}_{1}=\left[r_{1} / 2\right] w_{1}\left[\dot{x_{1}} / 2\right] w_{1}\left[\dot{x_{2}} / 2\right] w_{1} \ldots\left[\dot{x}_{\# 1-2} / 2\right] w_{1}\left[\ddot{x}_{\# 1-1} / 2\right]\left[t_{1} / 2\right] \\
\vec{B}_{1}=k_{1,1} w_{1} k_{1,2}
\end{gathered}
$$

Note that there are $\# 1-1$ words $w_{1}$ in $\vec{A}_{1}$. The word $\vec{B}_{1}$ reduces to $w_{1}$ since the sequence of 0 symbols $k_{1,1}$ and $k_{1,2}$ will become bounded after a finite number of $s$-rounds (Lemma 4 ). This allows for periodicity.

The parity of the length of each of the sequences $t_{1}\left[\dot{x}_{i} / 2\right] r_{t}$ in $\vec{A}_{1}$ is a determining feature in the ultimate 
behavior of $T$. Indeed, if there is at least one $i$ such that the sequence of 0 symbols $t_{1}\left[\dot{x}_{i} / 2\right] r_{t}$ in $\vec{A}_{1}$ is odd and the word $w_{1}$ preceding it is entered with shift 0 , the word $w_{1}$ following it will be entered with shift 1 and conversely. If there is no such sequence then all words $w_{1}$ will be entered with the same shift. Now, if there is an $i$ such that the sequences of 0 symbols $t_{1}\left[\dot{x}_{i} / 2\right] r_{t}$ in $\vec{A}_{1}$ has odd length then it follows that $T$ always has unbounded growth on any initial word that produces a word that contains at least one word $\vec{A}_{1}$. The reason for this is that the two possible words $\vec{C}_{2}$ and $\vec{D}_{2}$ that can then be produced after one $s$-round on $\vec{A}_{1}$ again contain at least one word $\vec{A}_{1}$ and one word $\vec{B}_{1}$. Indeed, the fact that there is at least one sequence of 0 symbols $t_{1}\left[\dot{x}_{i} / 2\right] r_{t}$ in $\vec{A}_{1}$ that has odd length implies that if the word $w_{1}$ preceding $\left[\dot{x}_{i} / 2\right]$ results in $\vec{A}_{1}$ then the word $w_{1}$ following it will result in $\vec{B}_{1}$ and vice versa. We then have that words $\vec{C}_{2}$ and $\vec{D}_{2}$ again result in the production of at least one word $\vec{C}_{2}$ or $\vec{D}_{2}$ (from $\vec{A}_{1}$ in $\vec{C}_{2}$ and $\vec{D}_{2}$ ) and at least one word $w_{1}$ (from $\vec{B}_{1}$ in $\vec{C}_{2}$ and $\vec{D}_{2}$ ). Thus, once either $\vec{C}_{2}$ or $\vec{D}_{2}$ are produced the word produced with each successive $s$-round on $\vec{C}_{2}$ or $\vec{D}_{2}$ always contains at least one word $\vec{C}_{2}$ or $\vec{D}_{2}$ and at least one additional $w_{1}$ as compared to the word from the previous $s$-round. This gives unbounded growth.

If there is no sequence of 0 symbols $t_{1}\left[\dot{x}_{i} / 2\right] r_{t}$ in $\vec{A}_{1}$ that has odd length, then $T$ produces either one of the following words after one $s$-round on $\overrightarrow{A_{1}}$ :

$$
\begin{aligned}
& \overrightarrow{A_{2}}=\left[r_{1} / 4\right] \vec{A}_{1}\left[\dot{x_{1}} / 4\right] \vec{A}_{1}\left[\dot{x_{2}} / 4\right] \ldots \vec{A}_{1}\left[\dot{x}_{\# 1-2} / 4\right] \vec{A}_{1}\left[\ddot{x}_{\# 1-1} / 4\right]\left[t_{1} / 4\right]
\end{aligned}
$$

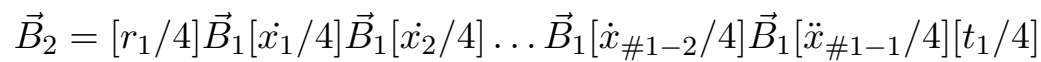

Again, if $\overrightarrow{A_{2}}$ contains at least one sequence of 0 symbols $t_{1}\left[\ddot{x}_{\# 1-1} / 2\right]\left[t_{1} / 2\right]\left[\dot{x}_{i} / 4\right]\left[r_{1} / 2\right] r_{1}, 0 \leq i \leq$ $\# 1-1$ that has odd length then it easily follows that $T$ always has unbounded growth on any word $A_{0}$ that results in the production of a word that contains $\vec{A}_{2}$ at least once. The reason is that the two possible words $\vec{C}_{3}$ and $\vec{D}_{3}$ that can be produced after one $s$-round on $\vec{A}_{2}$ each contain at least one word $\vec{A}_{2}$ and one word $\vec{B}_{2}$. Thus, the word produced with each new $s$-round on one of the words $\vec{C}_{3}$ or $\vec{D}_{3}$ must again contain at least one word $\vec{C}_{3}$ or $\vec{D}_{3}$ and at least one additional word $\vec{B}_{2}$. This results in unbounded growth.

Similarly, if there is at least one sequence of 0 symbols $t_{1} k_{1,2}\left[\dot{x}_{i} / 4\right] k_{1,1} r_{1}$ in $\vec{B}_{2}$ with odd length, then $T$ always has unbounded growth on any word $A_{0}$ that results in the production of a word that contains $\vec{A}_{1}$ at least once for similar reasons. Note that $\vec{B}_{2}$ is similar to $\vec{A}_{1}$. I.e., $\vec{B}_{2}$ also contains $\# 1-1$ words $w_{1}$. This allows for periodicity in the same way as the production of $\vec{B}_{1}$ does. The only difference between $\vec{A}_{1}$ and $\vec{B}_{2}$ is the number of 0 symbols separating consecutive words $w_{1}$ in $\vec{B}_{2}$. In this sense the possible productions from this word reduce to that of the word $\overrightarrow{A_{1}}$ and we will thus not consider these productions here.

In general we have two possibilities. In the first possibility there is an $n$ such that one of the words $\vec{A}_{n}$ or $\vec{B}_{n}$ given below exists. In the second possibility there exist no such words $\vec{A}_{n}$ or $\vec{B}_{n}$. The word $\vec{A}_{n}$ has the following form:

$$
\vec{A}_{n}=\left[r_{1} / 2^{n}\right] \vec{A}_{n-1}\left[\dot{x_{1}} / 2^{n}\right] \vec{A}_{n-1}\left[\dot{x_{2}} / 2^{n}\right] \vec{A}_{n-1}\left[\dot{x}_{\# 1-2} / 2^{n}\right] \vec{A}_{n-1}\left[\ddot{x}_{\# 1-1} / 2^{n}\right]\left[t_{1} / 2^{n}\right]
$$

where $A_{n}$ contains the following odd length subword consisting entirely of 0 symbols:

$$
t_{1}\left[\ddot{x}_{\# 1-1} / 2\right]\left[t_{1} / 2\right] \ldots\left[\ddot{x}_{\# 1-1} / 2^{n-1}\right]\left[t_{1} / 2^{n-1}\right]\left[\dot{x}_{i} / 2^{n}\right]\left[r_{1} / 2^{n-1}\right] \ldots r_{1} / 2 r_{1}, 0 \leq i \leq \# 1-1
$$

$B_{n}$ has the following form:

$$
\vec{B}_{n}=\left[r_{1} / 2^{n}\right] \vec{B}_{n-1}\left[\dot{x_{1}} / 2^{n}\right] \vec{B}_{n-1}\left[\dot{x_{2}} / 2^{n}\right] \vec{B}_{n-1}\left[\dot{x}_{\# 1-2} / 2^{n}\right] \vec{B}_{n-1}\left[\ddot{x}_{\# 1-1} / 2^{n}\right]\left[t_{1} / 2^{n}\right]
$$


where $\vec{B}_{n}$ contains the following subword consisting entirely of 0 symbols of odd length:

$$
t_{1} k_{1,2}\left[\ddot{x}_{\# 1-1} / 4\right]\left[t_{1} / 4\right] \ldots\left[\ddot{x}_{\# 1-1} / 2^{n-1}\right]\left[t_{1} / 2^{n-1}\right]\left[\dot{x}_{i} / 2^{n}\right]\left[r_{1} / 2^{n-1}\right] \ldots\left[r_{1} / 4\right] k_{1,1} r_{1}
$$

It easily follows from Lemma 4 that one can decide in a finite number of steps whether or not there exists such an $n$. Now, if there is such an $n$ then $T$ has unbounded growth on any word $A_{0}$ that results in the production of a word that contains $\vec{A}_{n}$ or $\vec{B}_{n}$ at least once, else $T$ halts or becomes periodic on $A_{0}$. Clearly, it can be decided in a finite number of rounds $j$ whether or not $T$ will produce a word $Q_{j}$ that contains $\vec{A}_{n}$ or $\vec{B}_{n}$. The reason for this is that it follows from Lemma 4 that there is an $m \in \mathbb{N}$ such that for any word $\vec{A}_{m+i}$ and $\vec{B}_{m+i}, i \in \mathbb{N}$ the length of any sequence of 0 symbols between two consecutive 1 symbols is bounded by some constant and it must be the case that $n \leq m$. One thus only has to wait at most $m+1$ rounds on $A_{0}$ to see whether or not $T$ will produce these words.

If there is no such $n$ then we again have that $T$ will either halt, become periodic or have unbounded growth on $A_{0}$. Also here it can be decided in a finite number of rounds of $T$ on $A_{0}$ whether or not $T$ will have unbounded growth on $A_{0}$ for similar reasons.

Case III.D.2. $w_{1}=r_{1} 1 \dot{x}_{1} 1 \dot{x}_{2} 1 \ldots 1 \dot{x}_{\# 1-1} 1 t_{1}\left(\# 1 \geq 3, w_{0}=0\right)$

For any tag system $T$ covered by this case, all 1 symbols in $w_{1}$ are separated by an odd number of 0 symbols. As a consequence, either zero or \#1 1 symbols are read during one $s$-round on $w_{1}$. After one $s$-round on $w_{1} T$ thus produces either a sequence of 0 symbols which ultimately results in the production of $\epsilon$ or the word:

$$
\overrightarrow{A_{1}}=\left[r_{1} / 2\right] w_{1}\left[\dot{x_{1}} / 2\right] w_{1}\left[\dot{x_{2}} / 2\right] w_{1} \ldots\left[\dot{x}_{\# 1-1} / 2\right] w_{1}\left[t_{1} / 2\right]
$$

Note that the word $\overrightarrow{A_{1}}$ contains $\# 1$ words $w_{1}$.

Now, if there is at least one sequence of 0 symbols $t_{1}\left[\dot{x}_{i} / 2\right] r_{1}$ that has even length then the case reduces to the previous Case III.D.1. I.e., for tag systems covered by Case III.D.1., either at least one word $w_{1}$ or at least two words $w_{1}$ are produced after one $s$-round on $w_{1}$. Similarly, for any tag system $T$ if the word $\vec{A}_{1}$ produced after one $s$-round on $w_{1}$ contains at least one sequence of 0 symbols $t_{1}\left[\dot{x}_{i} / 2\right] r_{1}$ that is even, either at least one word $\overrightarrow{A_{1}}$ or at least two words $\overrightarrow{A_{1}}$ are produced after one $s$-round on $\vec{A}_{1}$. The ultimate behavior of $T$ then depends on the evolution of the parity of the number of 0 symbols separating consecutive words $w_{1}$ in the words produced from $\vec{A}_{1}$. Note that for any initial word $A_{0}$, if after two rounds of $T$ on $A_{0} T$ does not produce a word $Q_{2}$ that contains $\vec{A}_{1}$ at least once, then $Q_{2}$ is a sequence of 0 symbols and thus $T$ will halt on $A_{0}$. Otherwise, $T$ will either become periodic or have unbounded growth on $A_{0}$. If there is no sequence of 0 symbols $r_{1}\left[\dot{x}_{i} / 2\right] t_{1}$ of even length in $\vec{A}_{1} T$ either produces a sequence of 0 symbols or:

$$
\overrightarrow{A_{2}}=\left[r_{1} / 4\right] \vec{A}_{1}\left[\dot{x_{1}} / 4\right] \overrightarrow{A_{1}}\left[\dot{x_{2}} / 4\right] \vec{A}_{1} \ldots\left[\dot{x}_{\# 1-1} / 4\right] \overrightarrow{A_{1}}\left[t_{1} / 4\right]
$$

Again, if there is at least one sequence of 0 symbols $t_{1}\left[t_{1} / 2\right]\left[\dot{x}_{i} / 4\right]\left[r_{1} / 2\right] r_{1}$ of even length then the case reduces to Case III.D.1. If this occurs and after three rounds of $T$ on $A_{0} T$ does not produce a word $Q_{3}$ that contains $\overrightarrow{A_{2}}$ at least once, then $Q_{3}$ is a sequence of 0 symbols and thus $T$ will halt on $A_{0}$. Otherwise, $T$ will either become periodic or have unbounded growth on $A_{0}$.

As is clear from these productions either there is or is no $n$ such that after $n s$-rounds of $T$ on $A_{0} T$ produces the word:

$$
\vec{A}_{n}=\left[r_{1} / 2^{n}\right] \vec{A}_{n-1}\left[\dot{x_{1}} / 2^{n}\right] \vec{A}_{n-1}\left[\dot{x_{2}} / 2^{n}\right] \vec{A}_{n-1} \ldots\left[\dot{x}_{\# 1-1} / 2^{n}\right] \vec{A}_{n-1}\left[t_{1} / 2^{n}\right]
$$


and $\vec{A}_{n}$ contains at least one sequence of 0 symbols $t_{1}\left[t_{1} / 2\right] \ldots\left[t_{1} / 2^{n-1}\right]\left[\dot{x}_{i} / 2^{n}\right]\left[r_{1} / 2^{n-1}\right] \ldots\left[r_{1} / 2\right] r_{1}$ that has even length. Note that $T$ either always produces a word $\vec{A}_{j}$ or a sequence of 0 symbols after $j s$ rounds on $w_{1}$, with $j \leq n$ if there is such an $n$ and $j \in \mathbb{N}$ if there is no such $n$.

Clearly, if there is such an $n$ then the case reduces to Case III.D.1. It then follows that given some initial word $A_{0}$, if after $n+1$ rounds of $T$ on $A_{0} T$ does not produce a word $Q_{n+1}$ that contains $\vec{A}_{n}$ at least once, then $Q_{n+1}$ is a sequence of 0 symbols and thus $T$ will halt on $A_{0}$. Otherwise, $T$ will either become periodic or have unbounded growth on $A_{0}$. If there is no such $n$ then the behavior of $T$ reduces to those subcases of Case III.C. 2 for which there is no $n$ such that the rightmost and leftmost 1 in the words $\vec{A}_{n-1}$ in $\vec{A}_{n}$ are separated by an even number of 0 symbols. I.e., $T$ will either halt or have unbounded growth on any initial word $A_{0}$ and this can be decided in a finite number of steps.

Given the proofs of Cases I-III Theorem 1 follows

\section{Discussion}

It might be very hard, if not impossible, to prove the solvability of those classes of tag systems that are closest to TS $(2,2)$, i.e., $\mathrm{TS}(2,3)$ and TS $(3,2)$. The class TS $(2,3)$ contains the example provided by Post, which is known for its complexity. The class TS $(3,2)$ contains a tag system that is capable to simulate the $3 n+1$ problem, a number theoretical problem that is still open [10]. As far as our experience goes, it seems that the methods used in the present proof cannot be used to prove these classes decidable. For example, consider the application of the table method to Post's example from Sec. 1.1. Still, the table method is a very useful and simple tool to study and prove certain properties of tag systems. This method can also be automated and thus used in computer-based research on tag systems. It also allows us to reveal the structure of the kind of words that can be produced by a given tag system $T$. As is clear from the proof of Theorem 1, the different kinds of structures found for tag systems from the class TS $(2,2)$, with $l_{\min }\left\langle v, l_{\max }>v\right.$ is very simple and predictable. It is exactly this simplicity of the structure of the words that can be produced by tag systems $T$ from this class that allows us to decide the two decision problem discussed here.

In recent years there has been a lot of research on non-standard models of computability. One example comes from the context of Turing machines, where the standard model was generalized by allowing an infinitely repeated word to the left and right of the input (weak Turing machines) and left or right of the input (semi-weak Turing machines). This generalization has made it possible to find smaller universal Turing machines than those known for the standard model (see e.g. [4, 22]). It would be interesting to extend this research to tag systems. One could, for example, consider (universal) tag systems that cannot halt, but always have unbounded growth. Let us call such (universal) tag systems weak (universal) tag systems. ${ }^{4}$ However, even if one considers such more general models, the simplicity of the structure of the words that can be produced by any tag system in the class $\operatorname{TS}(2,2)$, with $l_{\min }<v, l_{\max }>v$, seems

\footnotetext{
${ }^{4}$ Note that it is impossible to directly apply weak or semi-weak universality as defined for cellular automata and Turing machines to tag systems, because one always has to tag something at the end of a word, on the basis of what happens at the beginning of a word. One round on a word $A$ would then take infinite time.
} 
to exclude the possibility of universal encoding for any of these tag systems. ${ }^{5}$ In this sense, the proof of Theorem 1 gives strong support for the following conjecture:

Conjecture 1. The class of tag systems $\operatorname{TS}(2,2), l_{\min }<v, l_{\max }>v$ is not (weak) universal.

Given Wang's theorem (Sec. 1.1) we only had to consider tag systems with $l_{\min }\left\langle v, l_{\max }>v\right.$ to prove

Theorem 1 and thus we did not study the more general class, including those cases for which $l_{\min } \geq v{ }^{6}$ A more intensive analysis would be needed to know whether the following statement is true or false:

Statement 1. There exists no unsolvable decision problem for tag systems from the class TS(2,2).

If false, this could imply that there is weak universality in the class $\operatorname{TS}(2,2)$.

\section{References}

[1] Church, A.: An unsolvable problem of elementary number theory, American Journal of Mathematics, (58), 1936, 345-363, Also published in [5], 88-107.

[2] Cocke, J., Minsky, M.: Universality of Tag systems with P = 2, 1963, Artificial Intelligence Project - RLE and MIT Computation Center, memo 52.

[3] Cocke, J., Minsky, M.: Universality of Tag systems with P = 2, Journal of the ACM, 11(1), 1964, 15-20.

[4] Cook, M.: Universality in Elementary Cellular Automata, Complex Systems, 15(1), 2004, 1-40.

[5] Davis, M.: The Undecidable. Basic papers on undecidable propositions, unsolvable problems and computable functions, Raven Press, New York, 1965, Corrected republication (2004), Dover publications, New York.

[6] Davis, M.: Why Gödel didn’t have Church's thesis, Information and Control, 54, 1982, 3-24.

[7] Davis, M.: Emil L. Post. His life and work, 1994, In: [17], xi-xviii.

[8] De Mol, L.: Closing the circle: An analysis of Emil Post's early work., The Bulletin of Symbolic Logic, 12(2), 2006, 267-289.

[9] De Mol, L.: Study of limits of solvability in tag systems, Machines, Computations, and Universality. Fifth International Conference, MCU 2007 Orléans (J. Durand-Lose, M. Margenstern, Eds.), 4664, Springer, Berlin, 2007.

[10] De Mol, L.: Tag systems and Collatz-like functions, Theoretical Computer Science, 390(1), $2008,92-101$.

[11] Margenstern, M.: Frontier between Decidability and Undecidability: A survey, Theoretical Computer Science, 231(2), 2000, 217-251.

[12] Maslov, S. J.: On E. L. Post's 'Tag' Problem. (Russian), Trudy Matematicheskogo Instituta imeni V.A. Steklova, (72), 1964b, 5-56, English translation in: American Mathematical Society Translations Series 2, 97, 1-14, 1971.

[13] Minsky, M.: Recursive unsolvability of Post's problem of tag and other topics in the theory of Turing machines, Annals of Mathematics, 74, 1961, 437-455.

\footnotetext{
${ }^{5}$ Of course, the results of this paper prove that the class $\mathrm{TS}(2,2)$ cannot be universal in the standard sense as the system must have an unsolvable halting problem.

${ }^{6}$ To study the reverse cases with $l_{\text {max }} \leq v$ would not be interesting, since those cases always halt or become periodic.
} 
[14] Minsky, M.: Size and Structure of Universal Turing Machines using Tag systems: a 4-symbol 7-state machine, Proceedings Symposia Pure Mathematics, American Mathematical Society, 5, 1962, 229-238.

[15] Post, E. L.: Formal Reductions of the General Combinatorial Decision Problem, American Journal of Mathematics, 65(2), 1943, 197-215.

[16] Post, E. L.: Absolutely unsolvable problems and relatively undecidable propositions - Account of an anticipation, 1965, In: [5], 340-433. Also published in [17].

[17] Post, E. L.: Solvability, Provability, Definability: The collected works of Emil L. Post, Birkhauser, Boston, 1994, Edited by Martin Davis.

[18] Stillwell, J.: Emil Post and His Anticipation of Gödel and Turing, Mathematics Magazine, 77(1), 2004, 3-14.

[19] Turing, A. M.: On computable numbers with an application to the Entscheidungsproblem, Proceedings of the London Mathematical Society, (42), 1936-37, 230-265, A correction to the paper was published in the same journal, vol. 43, 1937, 544-546. Both were published in [5], 116-151.

[20] Wang, H.: Tag systems and lag systems, Mathematische Annalen, 152, 1963a, 65-74.

[21] Woods, D., Neary, T.: On the time complexity of 2-tag systems and small universal Turing machines, Proceedings of the 47th Annual IEEE Symposium on Foundations of Computer Science, 2006.

[22] Woods, D., Neary, T.: Small semi-weakly Universal Turing Machines, Machines, Computations, and Universality. Fifth International Conference, MCU 2007 Orléans (J. Durand-Lose, M. Margenstern, Eds.), 4664, Springer, 2007. 